\title{
Towards Advanced Electron Beam Brightness Enhancement and Conditioning
}

\author{
A white paper based on the \\ ANL Theory Institute on \\ Production of Bright Electron Beams \\ held at Argonne National Laboratory \\ September 22-26, 2003
}

K.-J. Kim, ANL (editor)

B. Carlsten, LANL

D. Dowell, SLAC

K. Flöttmann, DESY

K. Jensen, NRL

J. Petillo, SAIC

A. Sessler, LBNL

G. Stupakov, SLAC

February 2004 



\section{Table of Contents}

Executive Summary . 1

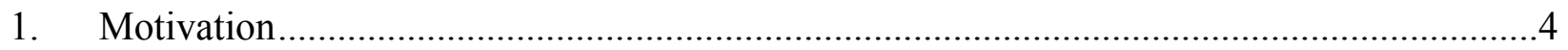

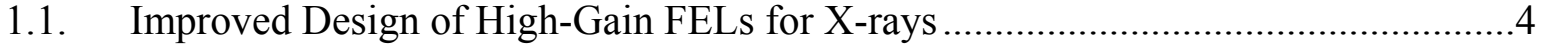

1.2. Application to Linear Colliders ………………...............................................

1.3. Impact on Other Projects ……………………….............................................

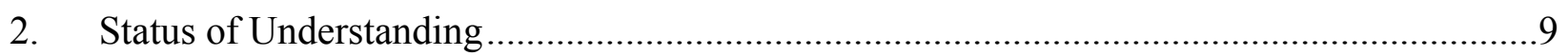

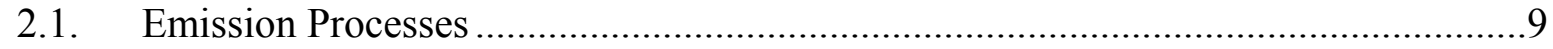

2.2. Beam Dynamics of Photocathode rf Guns..............................................................17

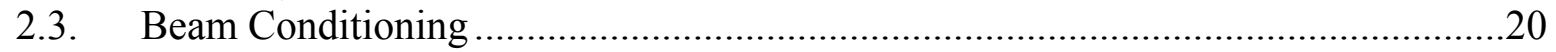

3. Outstanding Problems and Approaches for Their Solution ………………......................24

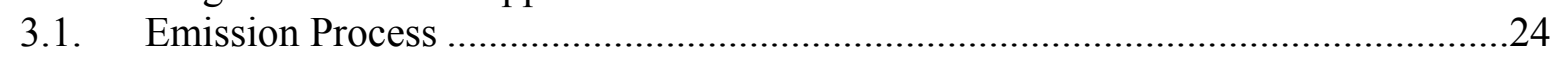

3.1.1. General Discussion of Goals..................................................................24

3.1.2. Summary of the Approaches for Emission Process Research and

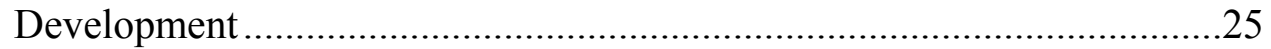

3.2. Beam Dynamics of $\mathrm{rf}$ Photocathode Guns............................................................2

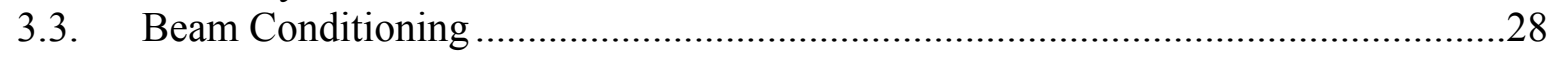

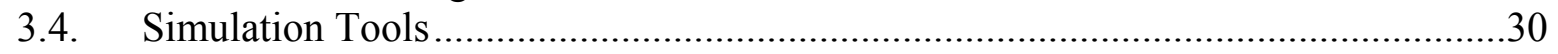

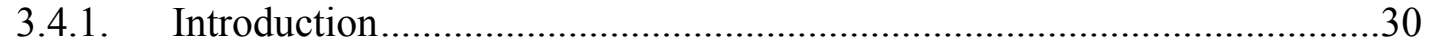

3.4.2. Development Plan ................................................................................

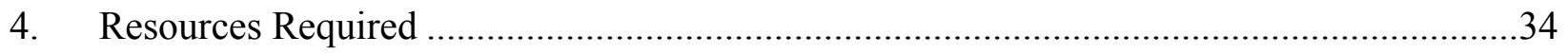

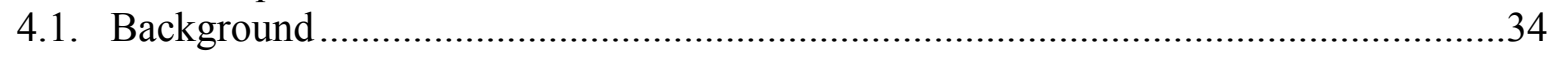

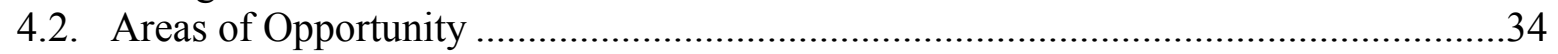

4.2.1. Properties of Cathode Materials and the Determinants of Intrinsic Emittance ............................................................................................

4.2.2. Early Stages of Acceleration (Guns and Space-Charge Effects in

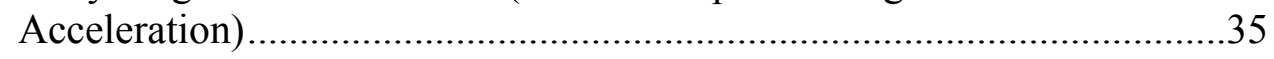

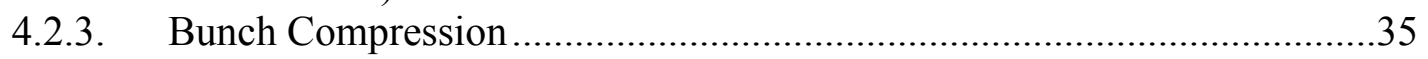

4.2.4. Other Advanced Techniques....................................................................36

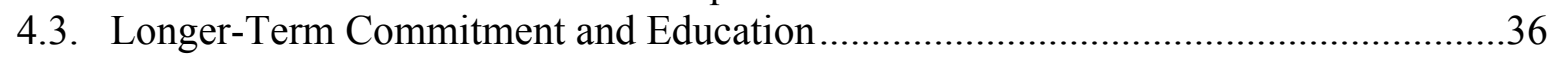

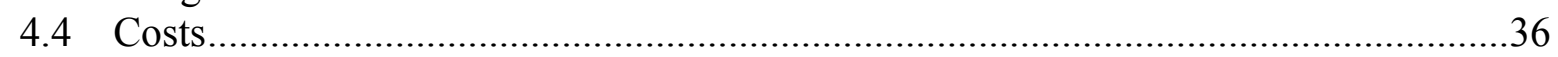

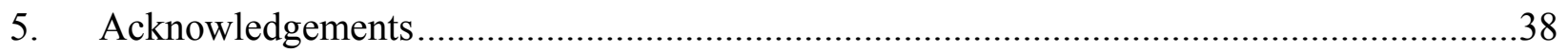

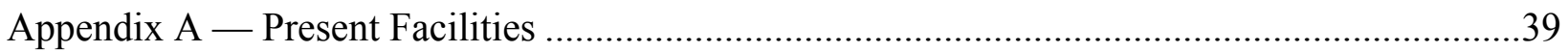

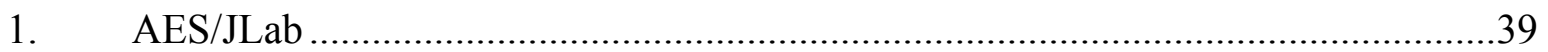

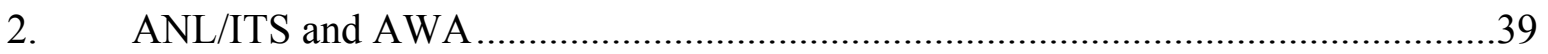

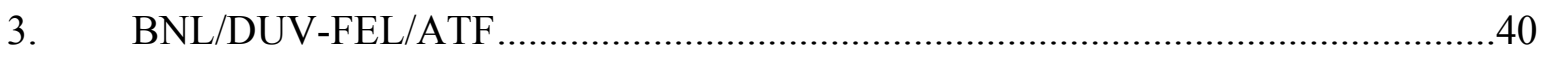

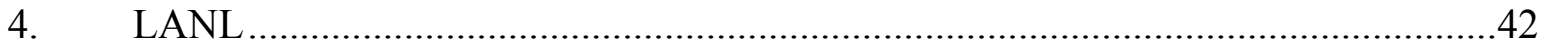

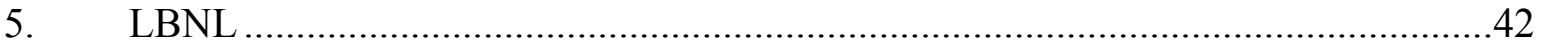

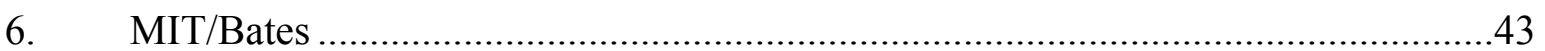

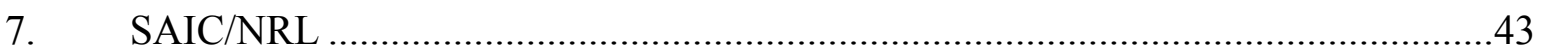

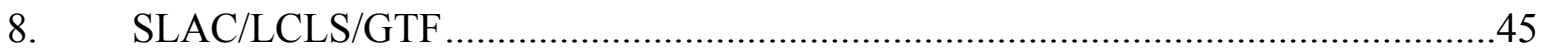

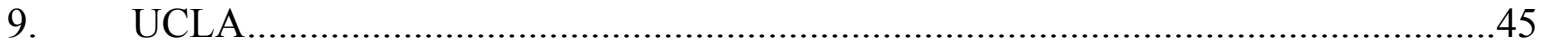




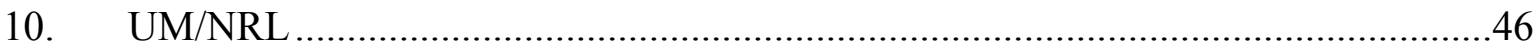

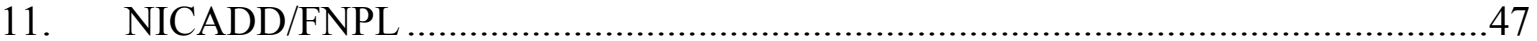

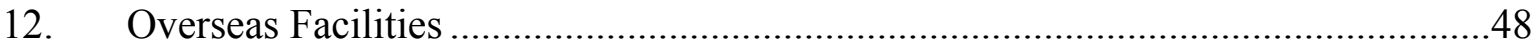

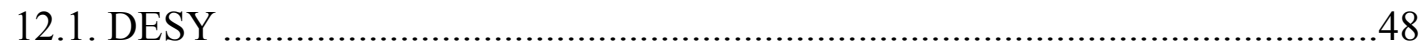

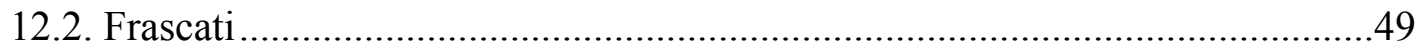

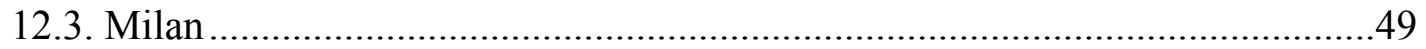

Appendix B - Program of the ANL Theory Institute on Production of Bright Electron

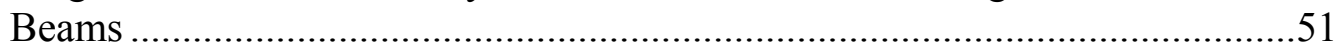

Appendix C - List of Participants at the ANL Theory Institute on Production of Bright Electron Beams. 


\section{Executive Summary}

The importance of developing higher-brightness electron beam sources for future accelerators was emphasized during the DOE-BESAC Subcommittee Meeting on the BES 20-Year Facility Roadmap held in Washington on February 20, 2003 [1]. The Subcommittee made a strong recommendation for an R\&D program for high-brightness gun development. Spurred by this recommendation, a workshop was organized at Argonne National Laboratory on September 2226, 2003 under the auspices of the ANL Theory Institute. About thirty experts in electron gun physics came for a week of intense discussion, reviewing the current state of the art and exploring ways to improve the performance of laser-driven rf photocathode guns, in particular whether an order of magnitude improvement of the beam brightness is feasible. Presentations given during the workshop can be found on the Internet at http://www.aps.anl.gov/asd/theory/ presentations-online.html.

This white paper grew out of the Argonne workshop. Its aim is to provide a summary of the ANL workshop and to propose an R\&D program to develop advanced electron sources with an order of magnitude higher brightness than currently feasible. The budget for the R\&D program for this purpose, discussed in Section 4, is estimated to be about \$10M per year for ten years with an additional onetime start-up cost of $\$ 10 \mathrm{M}$. Although expensive, the expenditure is well worthwhile in view of the tremendous benefits the higher brightness gun will bring in future accelerator development, in particular a fourth-generation light source based on X-ray freeelectron laser (FEL) technology and a future linear collider, which together will cost about \$10B. Facilities capable for general accelerator-based R\&D have been and are constructed as a part of larger accelerator complexes for research in various scientific disciplines, generally without provision for their use for research in advancing accelerator science. Sophistication in accelerator devices has become such that it is now necessary to promote accelerator research in its own right to be pursued with strong participation by university groups.

Section 1 contains the benefits of high-brightness electron beams. The two most prominent electron accelerator projects are a high-gain FEL for intense x-ray beams and a linear collider for high-luminosity electron-positron collisions at a center of mass energy of $500 \mathrm{GeV}$ or greater for elementary particle physics. Availability of higher-brightness electron sources would have a tremendous impact on these projects. For a linear collider, it opens the possibility of obviating the damping rings. For a high-gain FEL, it will make it possible to employ a shorter undulator for a given x-ray wavelength or to reach higher in the x-ray spectral range than feasible with the current sources. The performance of x-ray FELs can also be improved by a non-symplectic emittance exchange in which the transverse emittance is decreased at the expense of a larger energy spread or by beam conditioning, that is, introducing a correlation between the transverse and temporal distributions. In general, high-brightness electron beams show the promise of more compact and more versatile beam devices, and thus will also have impact in many areas in DOE missions other than BES and non-DOE areas, including electron microscope and streak camera development; extreme high-frequency, low-voltage rf sources; FELs for industrial and defense applications; and high-resolution radiography.

Section 2 is a review of the current understanding of the physics of electron beam generation and evolution. The emission process of laser photons producing electrons inside the cathode material, 
the electrons migrating to the surface, and subsequently emitting into vacuum is a complex phenomenon. Emission characteristics such as the response time, quantum efficiency, and the intrinsic emittance depend on the details of bulk and surface properties of the photocathode material. The electron beam produced at the cathode evolves as it propagates inside the rf cavity region under the influence of the rf and space-charge fields. The phase-space distribution of the beam emerging from the cavity exhibits correlations and distortions. These undesirable features can be minimized by shaping the laser pulse and introducing cavity harmonics and corrected to a certain extent with suitable beam optics manipulation. For FEL applications, the effective emittance can be also enhanced by advanced techniques such as beam conditioning and emittance exchange. Numerical simulation confirms that the emittance requirement for x-ray high-gain FELs is significantly relaxed by beam conditioning, i.e., by introducing a correlation between the transverse emittance and the energy spread. However, a practical scheme for conditioning has not been worked out yet. A relatively compact scheme employing two rf cavities and a solenoid between them results in an increase in the projected emittance, which is too large for $\mathrm{x}$-ray high-gain FELs. Gentler schemes involving rf cavities in combination of chromatic FODO lattice or TM110 rf cavities with sextupoles, on the other hand, turn out to be rather weak, perhaps requiring a ring arrangement.

In Section 3, approaches for solving outstanding problems in achieving brightness enhancement and conditioning are discussed. In the area of the emission process, the capabilities to compute the intrinsic emittance and quantum efficiency should be developed by building emission models from first principles. A focused experimental program should be developed to verify the emission models. Facilities should be constructed for testing new cathode designs. In the area of beam dynamics, new guns producing an order of magnitude higher brightness should be designed. Gun test facilities should be constructed making optimal use of the existing capabilities. In the area of beam conditioning or other beam manipulation techniques, effort to find a practical scheme should continue in view of its promise. Simulation codes should be refined to take into account the emission models and to allow a higher-precision calculation in beam dynamics. Specifically, there are needs to model the emission process, detailed interparticle behavior including the image charge effects, and fine structure and beam-edge details. Codes with large-scale parallel computing capabilities based on the latest EM PIC modeling methods should be developed.

Resources required for R\&D in electron gun development are discussed in Section 4. Technical infrastructure for accelerator R\&D should be constructed, maintained, and operated as user facilities in national laboratories open to university groups and others through competitive grants. A decade of sustained effort will be required to achieve the ambitious goal for an order of magnitude improvement in electron source brightness. Research disciplines required in the area of the emission process include surface chemistry and physics, laser techniques, nanoscale structures, and solid state physics. Two or three small, university-based research groups could make considerable progress. The beam dynamics area can be considered in two parts: the first part would address the early stage of acceleration involving beam evolution in an rf cavity under space-charge effects and the emittance compensation process, and the second part would address bunch compression. The first part requires access to high-voltage DC and/or rf sources, facilities for acceleration to 5-50 MeV, and a suite of beam diagnostics devices. An optimistic estimate of the required resources for this part is 4-6 research groups and two all purpose gun test facilities. 
The research on bunch compression, which was not explicitly discussed during the ANL meeting but is important in the discussion of the resources, would be very expensive, requiring bright electron beams ranging in energy from a few $\mathrm{MeV}$ to as high as a few $\mathrm{GeV}$. Experimental research on bunch compression should therefore be carried out primarily in national laboratories. However, university groups could make valuable contributions in areas such as diagnostics. In the area of advanced techniques such as beam conditioning, emittance exchange, and femtosecond and attosecond time scales, the activities at least for some time will be largely biased toward theoretical/computational studies. The budget for the activities to develop higherbrightness electron sources is estimated to be roughly $\$ 10 \mathrm{M}$ per year for ten years with a onetime start-up cost of roughly $\$ 10 \mathrm{M}$. The total cost of the program is therefore less than $5 \%$ of the projected cost of $\$ 1-2 \mathrm{~B}$ to construct a multiuser $\mathrm{x}$-ray user facility at a green site. It is also less than $1 \%$ of the combined cost of $\$ 10 \mathrm{~B}$ to construct an $\mathrm{x}$-ray user facility and a linear collider.

\section{Reference}

[1] BESAC Subcommittee Workshop Report on 20-Year Basic Energy Sciences Facilities Roadmap, February 22-24, 2003, http://www.sc.doe.gov/production/bes/BESAC/ 20year_facilities_report.pdf.. 


\section{Motivation}

Two of the most prominent future accelerator projects currently under discussion are a high-gain free-electron laser (FEL) for intense x-ray beams for material science research and a linear collider for electron-positron collisions at a center of mass energy of $500 \mathrm{GeV}$ or greater for elementary particle physics. High-brightness electron beam sources occupy central importance in both of these projects. Techniques to improve the state-of the-art in the brightness of the electron sources would have a tremendous impact in the future development of high-gain FELs and linear colliders in reducing the size and cost of these machines. There are also a number of other projects that will benefit from high-brightness electron sources.

\subsection{Improved Design of High-Gain FELs for X-rays}

High-gain FELs are proving themselves as research tools around the world. Attracted by the promise of a ten orders of magnitude increase in the peak brightness than that obtained in the third-generation facilities, new facilities are proposed to start operation around the end of this decade-LCLS in 2009 [1] and TESLA FEL [2] in 2012. These facilities are designed to reach the limits of FEL performance in x-ray wavelengths with currently known accelerator technology. Based on the successful operation of these first-generation facilities, one can expect that higher performance will be sought from these facilities, and more ambitious facilities will be designed and constructed sometime in the future [3]. Concepts and designs for higherperformance FELs will take advantage of the operational experience from the first-generation facilities, as well as R\&D progress in accelerator science and technology over the next five to ten years. In addition, the facilities now under construction (like LCLS) could, in an improvement program, take advantage of R\&D progress.

In particular, advances in electron gun techniques could have a significant impact in the design of future FELs. The current state-of-the-art electron sources - the rf photocathode gunsproduce bright beams of $1 \mathrm{nC}, 1 \mathrm{~mm}$-mrad transverse normalized emittance. On the other hand, the design and performance of high-gain FELs for sub-Angstrom wavelengths would be dramatically improved if one can achieve electron beam brightness about one order of magnitude smaller than the current state of the art, $0.1 \mathrm{~mm}$-mrad. Since the unnormalized emittance is inversely proportional to the energy, it is possible to partially offset the large normalized emittance by employing a higher energy electron beam than is necessary simply to satisfy the FEL resonance condition for producing radiation of a particular wavelength, as is done in the case of the LCLS and the TESLA FEL. Higher energy at a fixed wavelength also implies a higher deflection parameter $\mathrm{K}$, and hence a higher magnetic field, than is optimum. The optimum value of $\mathrm{K}$ is about 1.4 when other parameters are optimally chosen. This is illustrated for the LCLS case for 1.5 Angstrom in Table 1 and for the greenfield FEL for 0.4 Angstrom in Table 2. 
Table 1: Improvement of the 1.5-Å FEL with Low-Emittance Electron Beams

\begin{tabular}{|c|c|c|c|}
\hline $\begin{array}{c}\text { Electron } \\
\text { energy } \\
\mathbf{E} \\
(\mathrm{GeV}) \\
\end{array}$ & $\begin{array}{c}\text { Normalized } \\
\text { emittance } \\
\varepsilon_{\mathbf{n}} \\
(\mathbf{m m}-\mathbf{m r a d})\end{array}$ & $\begin{array}{c}\text { Deflection } \\
\text { parameter } \\
\text { K }\end{array}$ & $\begin{array}{c}\text { Saturation } \\
\text { length } \\
\text { L } \\
(\mathrm{m}) \\
\end{array}$ \\
\hline 14 & 1.2 & 3.7 & 84 \\
\hline 14 & 0.5 & 3.7 & 50 \\
\hline 14 & 0.1 & 3.7 & 29 \\
\hline 7 & 0.1 & 1.4 & 30 \\
\hline
\end{tabular}

The electron current of $3.5 \mathrm{kA}$, undulator period of $3 \mathrm{~cm}$, and beam envelope function $\beta_{\mathrm{x}}=18 \mathrm{~m}$ are kept fixed throughout this table. The first row corresponds to the LCLS case. The second and third rows show that by reducing the emittance to $\varepsilon_{\mathrm{n}}=0.5 \mathrm{~mm}-\mathrm{mrad}$ and $\varepsilon_{\mathrm{n}}=0.1 \mathrm{~mm}$-mrad, respectively, the saturation length is reduced to $50 \mathrm{~m}$ and $29 \mathrm{~m}$, respectively. The last row shows that the deflection parameter $\mathrm{K}$ can also be reduced to 1.4, and thus the electron energy is reduced to $7 \mathrm{GeV}$ while the saturation length remains practically the same as the case in the third row.

Table 2: Improvement of a 0.4- $\AA$ FEL with Low-Emittance Electron Beams

\begin{tabular}{|c|c|c|c|}
\hline $\begin{array}{c}\text { Electron } \\
\text { energy } \\
\mathbf{E} \\
(\mathrm{GeV}) \\
\end{array}$ & $\begin{array}{c}\text { Normalized } \\
\text { emittance } \\
\varepsilon_{\mathbf{n}} \\
(\mathbf{m m}-\mathbf{m r a d})\end{array}$ & $\begin{array}{c}\text { Deflection } \\
\text { parameter } \\
\text { K }\end{array}$ & $\begin{array}{c}\text { Saturation } \\
\text { length } \\
\mathbf{L} \\
(\mathrm{m}) \\
\end{array}$ \\
\hline 30 & 1.2 & 3.7 & 300 \\
\hline 30 & 0.5 & 3.7 & 130 \\
\hline 30 & 0.1 & 3.7 & 40 \\
\hline 14 & 0.1 & 1.4 & 60 \\
\hline
\end{tabular}

The undulator period of $3 \mathrm{~cm}$ and electron current of $3.5 \mathrm{kA}$ are kept fixed throughout this table. The first row shows that the saturation length of the FEL is $300 \mathrm{~m}$ if the electron beam and the undulator parameter are the same as in the LCLS except that the electron energy is $30 \mathrm{GeV}$. The next two rows show that the saturation length is dramatically reduced to $130 \mathrm{~m}$ and $40 \mathrm{~m}$, respectively, if the electron emittance could be reduced to $0.5 \mathrm{~mm}-\mathrm{mrad}$ or $0.1 \mathrm{~mm}-\mathrm{mrad}$, respectively. The last row demonstrates that a high-performance FEL can be designed with a $14-\mathrm{GeV}$ linac and significantly with a weaker magnetic field in which $\mathrm{K}=1.4$ if an electron gun producing an order of magnitude smaller emittance were available.

A gun producing ultralow emittance $(0.1 \mathrm{~mm}-\mathrm{mrad}), 1-\mathrm{nC}$ beams can probably be constructed. However, developing such a gun is a challenging task that would require intense R\&D efforts over several years to overcome the technical challenges associated with high accelerating field to 
minimize the space-charge-induced emittance growth; suitable cathode materials with minimum intrinsic emittance; and an integrated design for cathode, cavity, and focusing configuration to compensate the space-charge emittance. The ultralow-emittance guns will not be ready for the LCLS project due to the time scale involved. However they could have very significant impact on future FELs if the relevant R\&D can be launched now, and they could, of course, be of great benefit to LCLS and TESLA in future years.

There are other approaches to improve the FEL performance that are also sufficiently promising as to deserve R\&D. A very promising approach is the "beam conditioning," in which a suitable correlation is introduced between the electron energy and the amplitude of the betatron oscillation [4]. The conditioning minimizes the spread of the forward velocity of electrons so that the FEL remains in resonance for a longer time. The effect of conditioning on the evolution greenfield FEL as a function of the distance along the undulator is shown in Figure 1 [5]. It is seen that the conditioning improves the FEL performance significantly, in particular with a stronger focusing. A practical design of a conditioning system is challenging due to the nonlinear nature of the problem. However, some promising schemes were suggested during the Workshon

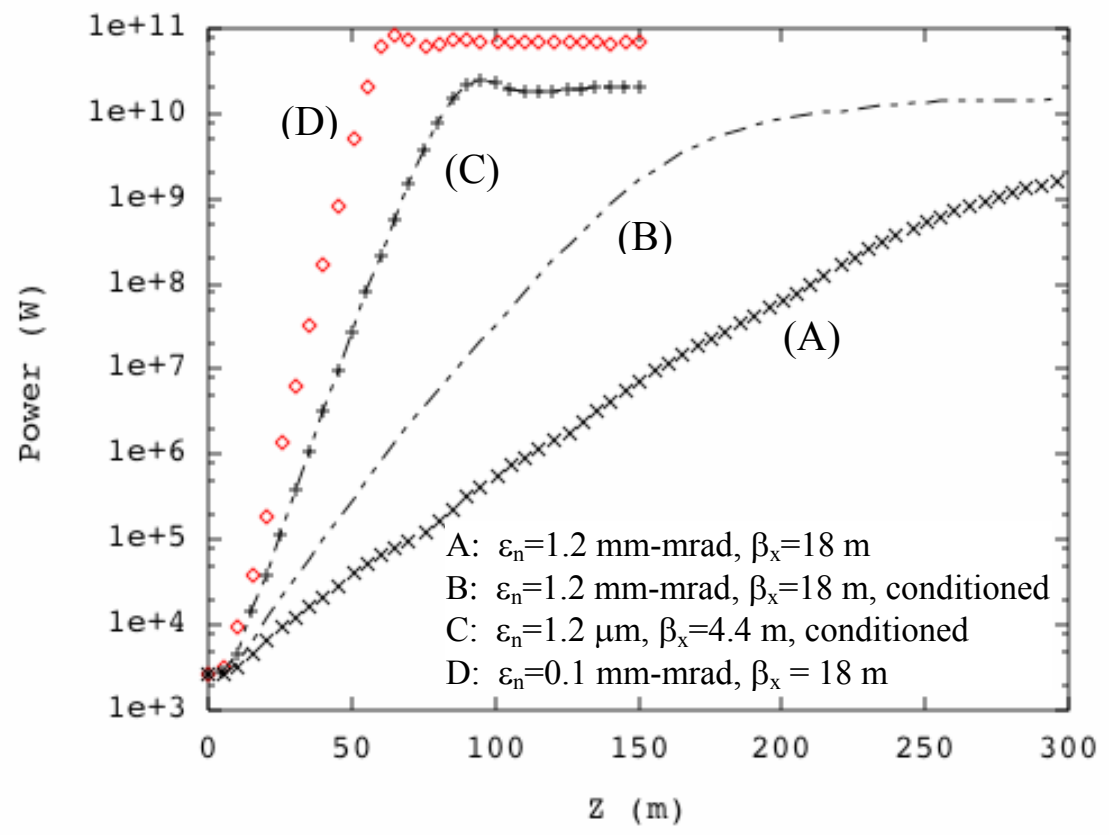

Figure 1: Effect of conditioning on the performance of an FEL. Curve (A) is for unconditioned beams with $\varepsilon_{\mathrm{n}}=1.2 \mathrm{~mm}-\mathrm{mrad}$, corresponding to the first row in Table 2. Curve (B) shows the improvement of the FEL gain when the beam is conditioned. Curve (C) shows that improvement is more dramatic when the beam conditioning is combined with a stronger focusing, $\beta_{\mathrm{x}}=4.4 \mathrm{~m}$. Finally, curve (D) is for an unconditioned beam but with $\varepsilon_{\mathrm{n}}=0.1 \mathrm{~mm}-\mathrm{mrad}$. From Ref. [5].

The FEL performance can also be improved if a portion of the transverse emittance can be traded with the longitudinal emittance. The electron energy spread at the entrance of the undulator is $\Delta \mathrm{E} / \mathrm{E}<10^{-5}$, which is two orders of magnitude smaller than the requirement for the high-gain FEL $\Delta \mathrm{E} / \mathrm{E}<10^{-3}$. Thus we have the situation that the transverse phase-space area is too large 
while the longitudinal phase-space area is too small for an optimal FEL operation for subAngstrom wavelengths. The mismatch may be corrected by an area-preserving transformation from $\varepsilon_{\mathrm{nx}} \quad \varepsilon_{\mathrm{ny}} \Delta \mathrm{E} / \mathrm{E}=(1 \mathrm{~mm}-\mathrm{mrad})^{2} 10^{-5}$ to $\varepsilon_{\mathrm{nx}} \varepsilon_{\mathrm{ny}} \Delta \mathrm{E} / \mathrm{E}=(0.1 \mathrm{~mm}-\mathrm{mrad})^{2} 10^{-3}$. Such a transformation will also help to alleviate the harmful effects of small energy spread for accelerator operation, such as the coherent synchrotron radiation instability in the bunch compression chicanes [6]. Unfortunately, however, it is known that such a transformation is not possible in a symplectic Hamiltonian system [7]. It would be interesting to study whether a nonHamiltonian process involving, for example, radiation damping can be exploited to effect the desired transformation.

\subsection{Application to Linear Colliders}

A linear collider with $\mathrm{CM}$ energy of $500 \mathrm{GeV}$ or greater has been endorsed by the U.S. high energy physics community as the highest priority after the LHC construction. Electron guns producing ultralow emittance may obviate the damping rings in future linear colliders. The normalized emittances in the two transverse dimensions for the case of NLC are: $\varepsilon_{\mathrm{nx}}=3.6 \mathrm{~mm}$ $\operatorname{mrad}$ and $\varepsilon_{\mathrm{ny}}=0.04 \mathrm{~mm}-\mathrm{mrad}$, with the product $\varepsilon_{\mathrm{nx}} \varepsilon_{\mathrm{ny}}=0.14(\mathrm{~mm}-\mathrm{mrad})^{2}$ [8]. Such a beam may be created by an $\mathrm{rf}$ photocathode gun with $\varepsilon_{\mathrm{n}}=0.37 \mathrm{~mm}$-mrad combined with the flat-beam generation technique [9]. Of course there are issues associated with the polarization and positrons.

\subsection{Impact on Other Projects}

High-brightness electron beams have a surprisingly wide impact. In addition to DOE BESsponsored light sources, the development of the advanced sources of electron beams tailored to optimize high brightness have applications in other DOE missions, Department of Defense (DoD) missions, and commercial missions. Not surprisingly, much of the beam physics that is important for an X-ray FEL is also key for other bright electron beam applications, although there are differences. In general, producing extremely bright electron beams and conditioning them will decrease the size and cost for these applications. In some applications, the requirements are even stronger, because the proposed devices require the higher brightness to operate, independent of beam energy.

Electron microscopes would benefit from the availability of low-emittance electron sources, with charge and energy tunability, as provided by rf guns. Future generations of streak cameras with ultrahigh resolution would evolve following the development of rf systems tightly synchronized to laser oscillators - for guns the laser produces the electron bunch at a well-defined rf phase, for streak cameras the rf streak voltage phase is well-defined with respect to the sample excitation laser. Electron sources for high-energy physics will benefit from the development of reliable synchronized laser and rf systems, and laser pulse temporal and spatial profiling, for highly reproducible beams. The success of a new generation of extremely high-frequency (100-300 $\mathrm{GHz})$, low-voltage (20-120 kV) rf sources based on extending traveling-wave tube technology with the combination of sheet electron beams and microfabricated slow-wave structures depends on high-brightness electron beam physics. These devices are designed to include transverseplane emittance conversion, and will not operate with beam emittances (in the sheet beam's narrow dimension) greater than 0.25 to $0.5 \mathrm{~mm} \mathrm{mrad}$. 
Directly related to the DOE light source application is the FEL mission for the DoD, for shipboard missile defense. Increases in performance due to higher beam brightness and potential decreases in accelerator size are important on ships where size and weight must be traded off with other critical cargo. The same argument can be made for industrial uses for FELs such as the electron beam welding machines; they are commercially more attractive as the price drops and performance increases.

The beam physics that lead to high-brightness electron beams in rf guns and photoinjectors is very similar to that which dominates beam brightness in modern induction linacs, such as those used for DOE radiographic missions. Future DOE electron-beam-driven gamma-ray sources for both static and dynamic radiography will need better resolution and have smaller accelerators, both enabled by high-brightness electron beam research as proposed here.

\section{References}

[1] M. Cornacchia et al., Linac Coherent Light Source (LCLS) Design Study Report, SLAC-R521, 1998.

[2] G. Materlik and Th. Tschentscher, "The X-ray Free Electron Laser," TESLA Technical Report Part V, http://tesla.desy.de/new_pages/TDR_CD/PartV/fel.html.

[3] J. Galayda, K.-J. Kim, and J. Murphy, Greenfield FELs, BESAC Subcommittee on BES 20 Year Facility Road Map, Feb. 2003.

[4] A.M. Sessler, L.H. Yu, and D.H. Whittum, Phys. Rev. Lett. 68, 309 (1992).

[5] A. Wolski, G. Penn, A. Sessler, J. Wurtele, "Beam Conditioning for FELs: Consequences and Methods," LBNL-53899, CBP Note-544, October 2003.

[6] E. Saldin et al., "Longitudinal space charge driven microbunching instability in TTF2 Linac,” DESY-TESLA-FEL-2003-02, May 2003.

[7] E.D. Courant and H.S. Snyder, Ann. Phys. 3, 1 (1953).

[8] International Linear Collider Technical Review Committee, Second Report, SLAC publication (2003).

[9] Ya. Derbenev, "Adapting Optics for High Energy Electron Cooling," U. of Michigan report UM-HE-98-04, 1998. 


\section{Status of Understanding}

\subsection{Emission Processes}

\section{Introduction}

Currently, photocathode/drive laser combinations are capable of producing 1-nC bunches of a few picosecond duration with 1-2 mm-mrad emittance. If the emittance can be reduced by an order of magnitude from its present minimum, the design of an x-ray free electron laser can be simplified with a shorter linac and a shorter undulator. Obtaining an ultralow-emittance electron beam therefore has financial as well as scientific interests. In addition to emittances, the electron guns must be robust and shall satisfy suitable requirements for applications, quantum efficiency, and promptness of emission A concerted effort is required to achieve this challenging goal, requiring a new look at the photocathode process and the coupling of experiment and development with theory and simulation support.

\section{Emission Physics}

The relationship between emitted current and incident laser power in a photocathode can be represented as

$$
I=\left(\frac{q}{\hbar \omega}\right) \times P \times Q E,
$$

where $q$ is the electron charge, $\omega$ is the laser frequency, $P$ is the laser power, and $Q E$ is the quantum efficiency. In convenient units, this becomes

$$
I[\mathrm{~mA}]=\frac{1}{124} \lambda[\mathrm{nm}] P[\mathrm{~W}] Q E[\%]
$$

where $\lambda$ is the laser wavelength. Predicting $Q E$ constitutes a substantial challenge, and the approximations that are brought to bear on its estimation likewise impact predictive estimates of emittance and energy distributions. One-dimensional theory for metals suggests that asymptotically,

$$
Q E \propto\left\{\begin{array}{cc}
(\hbar \omega-\phi)^{2} & \phi \leq \hbar \omega \\
J_{R L D}(T, \phi-\hbar \omega) & \phi>\hbar \omega
\end{array},\right.
$$

where $\phi$ is the emission barrier height (e.g., work function $\Phi$ lowered by a Schottky factor due to applied field), $J_{R L D}$ is the Richardson coefficient for thermal emission, and $T$ is the temperature. Such an equation gives an indication of what factors affect emission, but it is evidently incomplete and provisional (for $\hbar \omega \approx \phi$, it is, moreover, incorrect). Nevertheless, it indicates that the desire to use longer wavelengths based on drive laser considerations is at odds with the desire to maximize $Q E$ using shorter wavelengths, unless the barrier can be reduced or circumvented. 


\section{Emittance Optimization}

The normalized rms emittance is defined as

$$
\varepsilon_{n}=\frac{1}{m c} \sqrt{\left\langle x^{2}\right\rangle\left\langle p_{x}^{2}\right\rangle-\left\langle x p_{x}\right\rangle^{2}}
$$

where $x$ and $p_{x}$ are the transverse coordinate and momentum in the x-direction, respectively, $<\ldots>$ indicates an average over particle distribution, $m$ is the electron mass, and $c$ is the speed of light. Postemission, a number of mechanisms contribute to emittance growth, such as spacecharge effects, time-dependent rf fields, nonlinear focusing, wakefields, and coherent synchrotron radiation in bends. However, the concern here is with regard to emittance contributions due to the material properties of the cathode, which may be referred to as the intrinsic emittance. Cathode emittance will depend on several factors, namely, the energy spread of the emitted electrons, their effective temperature, patchiness or nonuniformity of emission, surface roughness (which will give rise to both geometrically enhanced emission due to the Schottky effect and introduce transverse momentum components at the emission site), impurities, grain boundaries, and so on. A prediction of the emittance at the cathode is therefore predicated upon an adequate emission model, and will therefore be affected by the same factors that complicate the estimation of quantum efficiency.

In light of the aforementioned complications, it is clear that a predictive theory of photoemission is difficult: the physics near the surface of the cathode is critical but incompletely described; heuristic and one-dimensional accounts are provisional at best; and simulation codes using, for example, modified Richardson equations, in the case of thermal emission, or Fowler Nordheim equations, in the case of field emission, are necessarily incomplete. Nevertheless, it is believed that a coordinated effort by several groups over several years will give rise to simulation codes incorporating correct emission physics in such a way that predictive estimates of intrinsic emittance and quantum efficiency are feasible, and that such a process amongst particular groups has already begun.

The combination of (achievable) external fields, cathode materials, drive lasers, and operating conditions that will result in the maximum charge density in 6-D phase space from a zeroemittance source will depend on whether static or time-dependent fields are utilized. For a CW source, it is not clear whether DC or rf (particularly at room temperature, where fields are limited by thermal considerations) is better. The preference for one over the other will depend on the application and the bunch charge required, as applications that require high bunch charges but low repetition rate differ from $\mathrm{CW}$ pulse trains, which demand less bunch charge.

\section{Optimum Photocathodes}

Photocathode performance is dictated and characterized by a relatively small number of parameters to achieve useful emission, namely, bulk temperature, field strength at the cathode surface, work function at the emission site and/or the nature of coatings or contaminants, emission temporal response, and drive laser illumination particulars such as intensity, duration, shape, and pulse repetition frequency (duty factor). Fields on the cathode are generally 10 to 100 $\mathrm{MV} / \mathrm{m}$, and generally the cathode is desired to produce $0.1-1 \mathrm{nC}$ in FWHM pulse widths of $10 \mathrm{ps}$ 
in a disk approximately $1 \mathrm{~mm}^{2}$ in area. Note that $1 \mathrm{nC} / 10 \mathrm{ps}$ corresponds to 100 -A peak current, and the field produced by a sheet with a charge density of $\left(1 \mathrm{nC} / 1 \mathrm{~mm}^{2}\right)$ is $\mathrm{Q} /\left(2 \varepsilon_{0} \mathrm{~A}\right)=56.5$ $\mathrm{MV} / \mathrm{m}$, so space-charge effects within the disk can be disruptive.

Properties of different types of photocathodes are summarized in Table 3. Metallic photocathodes face problems for high average power machines, as the drive laser poses a challenge, and at high average current, the metal surface is damaged whether or not a suitable laser is available. Typical laser energies of 5 to $500 \mu \mathrm{J} /$ pulse are required for metal photocathodes, as compared to $0.5 \mu \mathrm{J} /$ pulse for semiconductor photocathodes. Metal cathodes have greater tolerance to vacuum conditions but require UV illumination and have lower quantum efficiencies (generally less than $0.01 \%$ ). For low duty factor operation, low repetition rate UV optical pulses can be obtained by frequency-quadrupled Nd lasers or tripled Ti:sapphire lasers. Metallic photocathodes have a fast response time, such that the femtosecond structure on the drive laser will appear on the electron beam.

All high quantum efficiency photocathodes are direct band-gap p-type semiconductors and belong to one of three families (i) alkali antimonides $\left(\mathrm{Cs}_{3} \mathrm{Sb}, \mathrm{K}_{2} \mathrm{CsSb}\right.$, etc.), which operate in the visible; (ii) alkali tellurides ( $\mathrm{Cs}_{2} \mathrm{Te}, \mathrm{KCsTe}$, etc.), which operate in the UV and near UV; and (iii) a number of bulk III-V semiconductors that use a surface dipole layer formed by Cs plus an oxidant like $\mathrm{O}$ or $\mathrm{F}$ and operate in IR through visible. The first two families have positive electron affinity (PEA) and have only been applied to rf guns, whereas the last shows negative electron affinity (NEA) and has only been used in DC guns. PEA photoemittters present a barrier at the surface to electron emission, whereas NEA cathodes do not (the conduction band lies above and below the vacuum level, respectively). Thus, a very low thermal emittance from NEA photoemitters may be possible because a reduction in cathode temperature results in a lower transverse temperature for the emitted electrons: the price paid is that the emission time is comparatively long (on the order of 10-20 ps).

The first two families require higher harmonics of the drive lasers. All are chemically reactive and thus sensitive to vacuum conditions, for example, being easily poisoned by $\mathrm{H}_{2} \mathrm{O}$ and $\mathrm{CO}_{2}$. Protecting these cathodes from such gases has been met with limited success and is generally at the significant expense of quantum efficiency. Even chemically "harmless" gases such as $\mathrm{H}_{2}$ and $\mathrm{CH}_{4}$ can be ionized by the emission beam and damage the cathode surface by ion back bombardment (which may affect the NEAs differently than the PEAs), and is a greater issue in DC guns. 
Table 3: Photocathode Candidates

(adapted from Z.M. Yusof, High Energy Physics Division, ANL)

\begin{tabular}{|c|c|c|}
\hline PHOTOCATHODE & GOOD & BAD \\
\hline $\begin{array}{l}\text { Metallic } \\
\text { (Cu, Mg, Ag, etc.) }\end{array}$ & $\begin{array}{ll}\text { - } & \text { Easy to obtain/handle } \\
\text { - } & \text { Widely-used } \\
\text { - } & \text { Rugged, and does not require } \\
\text { - } & \text { QHV constant for long time } \\
\text { - } & \text { Fast response time } \\
\text { - } & \text { Allows for pulse shaping }\end{array}$ & $\begin{array}{ll}\text { - } & \text { Low QE }(<0.01 \%) \\
\text { - } & \text { No systematic study of } \\
\text { effective cleaning \& } \\
\text { rejuvenating method, } \\
\text { especially in-situ at } \\
\text { photoinjector } \\
\text { - Not indicated for high average } \\
\text { power applications } \\
\text { - Beam tracks temporal } \\
\text { fluctuations in drive laser }\end{array}$ \\
\hline $\begin{array}{l}\text { PEA Semiconductor } \\
\left(\mathrm{Cs}_{2} \mathrm{Te}, \mathrm{K}_{2} \mathrm{Te}, \mathrm{GaN} \text {, }\right. \\
\text { etc.) }\end{array}$ & $\begin{array}{ll}\text { - } & \text { High QE (5-30\%) } \\
\text { - } & \text { Photoelectrons have lower } \\
\text { energy spread (in principle) } \\
\text { than metallic } \\
\text { - } \quad \text { Low dark currents }\end{array}$ & $\begin{array}{ll}\text { - } & \text { Requires UHV } \\
\text { - } & \text { Surface deteriorates with } \mathrm{O}_{2} \\
\text { - } & \text { Longer response time than } \\
& \text { metallic } \\
\text { - } & \text { Initial QE has short lifetime }\end{array}$ \\
\hline $\begin{array}{l}\text { NEA } \\
\text { (GaAs family, GaP, } \\
\text { etc.) }\end{array}$ & $\begin{array}{ll}\text { - } & \text { High QE }(10-60 \%) \\
\text { - } & \text { Widely used in PMT } \\
\text { - } & \text { Possible source of polarized } \\
& \text { electrons (GaAs) } \\
\text { - } & \text { Slow emission time damps } \\
& \text { laser fluctuations }\end{array}$ & $\begin{array}{ll}\text { - } & \text { Requires UHV } \\
\text { - } & \text { Even longer response time } \\
\text { than semiconductor } \\
\text { - } \\
\text { Difficult to use in an RF gun }\end{array}$ \\
\hline $\begin{array}{l}\text { "Needle" metal } \\
\text { cathodes }\end{array}$ & $\begin{array}{ll}\text { - } & \text { High brightness } \\
\text { - } & \text { QE (compared to metal) } \\
\text { enhanced by Schottky effect } \\
\text { - } \quad \text { Table-top source }\end{array}$ & $\begin{array}{l}\text { - Not clear that it can scale up } \\
\text { - Lack of repair in situ }\end{array}$ \\
\hline Dispenser Cathodes & $\begin{array}{l}\text { - } \quad \text { Low work function / High QE } \\
\text { - Self-repairing \& Robust } \\
\text { - } \quad \text { Mature technology base }\end{array}$ & $\begin{array}{l}\text { - Recent innovation - } \\
\text { qualification not available } \\
\text { (but underway). }\end{array}$ \\
\hline
\end{tabular}

Photocathodes such as GaAs have 10-ps scale time which, while insensitive to hash on the laser pulse, is insufficiently responsive for pulse shaping. A hypothetical cathode with a response time of $1 \mathrm{ps}$ would be fast enough for desired pulses and pulse shaping, but slow enough to suppress high-frequency structure on the pulse. While off-loading cathode response time issues onto the drive laser may be possible, it too is a complex problem.

Initial measurements of intrinsic emittance have been performed for GaAs (Dunham, et al., PAC95 at Dallas) and $\mathrm{Cu}$ (Graves, et al., PAC01 at Chicago). These should be repeated for other candidates $\left(\mathrm{Cs}_{2} \mathrm{Te}, \mathrm{Mg}\right.$ and $\left.\mathrm{Cu}, \mathrm{KCsSb}\right)$ and at other facilities (e.g., DESY, SLAC, BNL) and the results compared to theory and simulation. 
Concisely, the optimal photocathode is affected by an interplay between material parameters and drive laser specifics. The best photocathode and gun types are application dependent and are dictated by, for example, the repetition rate and pulse train duration, bunch charge, drive laser, emittance tolerances, and so on. The photocathode will require either a good vacuum or the ability to be self-cleaning or self-rejuvenating. Its emission characteristics will be dictated by the local work function (due to contaminants, coatings, surface geometry), which affects quantum efficiency, emission uniformity, local current density, and emittance, and impacts whether there are thermal and field emission components (dark current). Its performance relates to the optimal wavelength, emission promptness, and absorbed laser power, which in turn depends on material parameters such as band structure, electron scattering rates, reflectivity, laser penetration depth, thermal conductivity, and specific heat.

\section{Drive Lasers}

Using drive lasers with wavelengths longer than UV would have several advantages, e.g., efficiency, fluctuations, etc. Using a UV drive laser with a metallic cathode would preclude a reliable FEL with a high duty factor. High duty factors at higher harmonics result in heating problems in the crystals used to create the higher harmonics, as the efficiency of conversion decreases with higher harmonics.

Improvements in laser uniformity (cathode imperfections - in the form of field enhancement from roughness, impurities, grain boundaries, etc.) and laser nonuniformity conspire to produce hot spots. Tailoring of the laser beam profile may assist in overcoming space-charge effects, which are detrimental to emittance by enabling a stable longitudinal/transverse distribution in the high space-charge limit.

Laser pulse shape also has an impact on emittance. A top-hat ("beer can")-like profile is optimal: the space charge forces are much smaller than for a Gaussian beam. Profiles in which the charge density is uniform both transversely and longitudinally are best, with a similar demand on the pulse profile of the drive laser. However, longitudinal space-charge waves propagating from the ends of the pulse may adversely affect emittance. Lasers typically used produce quasi-Gaussian (or worse) profiles, which are altered when optical harmonic generation is used. Techniques for longitudinal pulse shaping generally aim towards shorter pulses than desirable for photoemission sources. The generation of optical pulses or pulse trains with transverse and longitudinal profiles to minimize electron beam emittance is an important area of research.

Low peak currents avoid emittance growth because of space charge. Such efforts to reduce emittance, by emitting long pulses that are subsequently compressed (which generates problems of its own and is not a simple procedure), can be at odds because higher peak currents enable smaller emission areas and reduce the impact of temporal variations in the rf field. SLAC is working on a third-harmonic gun that will allow longer pulses.

\section{Polarized Beams}

The high-energy physics community would potentially benefit from enabling reliable polarized electron sources. The generation of polarized beams at present exclusively relies on GaAs and 
related semiconductor photocathodes in DC guns. Efforts focus on obtaining cathodes that deliver the highest polarization, in excess of $80 \%$ at present, and possibly $90 \%$ for the best cathodes from SLAC.

NEA photocathodes benefit from a reduction in cathode temperature, as lower transverse $\mathrm{kT}$ results in reduced emittance. The down side is that such photocathodes are characterized by long emission times.

The use of a GaAs photocathode in an $\mathrm{rf}$ gun has resulted in failure within an hour after a short number of rf pulses. Both the technology base and the number of R\&D researchers is small.

\section{Outstanding Issues}

The small number of theoretical groups participating is a concern. A second theoretical group is needed, as are dedicated material scientists who understand the emission process and can, consequently, utilize that expertise to design appropriate photocathodes, whose properties are dictated by the applications for which they are intended.

Further, more basic science measurements are needed, and they need to be correlated with gun measurements. Support from laboratory administrations to perform measurements is essential. Some of the issues, described below, are in fact better suited for universities, in which case, the agenda needs to be tilted towards basic research, and the university groups need to be convinced that such work is important.

More sophisticated semiconductor photocathodes need to be developed and their properties quantified in order to improve them (using, e.g., angle-resolved photoemission and surface analysis techniques). Reliable methods must be developed for generating uniformly populated optical pulses to generate uniform charge distributions from the cathode to minimize emittance.

Emission models need to be applied to the estimation of quantum efficiency, intrinsic emittance, and emission distributions. The prediction of these models requires validation by comparison to existing experiments. What other predictions the models can make and what experiments can provide validation need articulation.

The performance of the gun is affected by material properties and processing of the surface; these should be systematically investigated. Where theoretical values of thermal emittance are not obtained, the reasons why should be investigated.

The 6-D phase space and intrinsic emittance at the cathode needs to be mapped and compared to the 0.1 micron goal - the lower limit on thermal emittance has downstream consequences on design and the nature of the electron bunch required (e.g., long vs. short, etc.), and specification of the conditions at the cathode are therefore critical.

Measurement and simulation of the intrinsic emittance is quite important ("intrinsic" rather than "thermal" to account for surface roughness, cathode type, and material). Does the quantum efficiency evolve in parallel with the intrinsic emittance? 


\section{Selected References}

\section{Electron Emission Pertaining to or Relevant to Photoemission}

- R.H. Fowler, Phys. Rev. 38, 45 (1931). (Canonical reference)

- L.E. DuBridge, Phys. Rev. 43, 727 (1933). (Canonical reference)

- C. Herring and M.H. Nichols, Rev. Mod. Phys. 21, 187 (1949). (While for thermionic emission, this encyclopedic review discusses many issues that affect photoemission, such as surface conditions, work function, field enhancement, etc.)

- H. Sommer, Photoemissive Materials, (Robert E. Krieger Publishing Company, Huntington, New York, 1980). (Standard reference.)

- H. Timan, "A Survey of Recent Advanced in the Theory and Practice of Vacuum Photoemitters," in Advances in Electronics and Electron Devices, (New York: Academic Press, 1985) 63:73. (Recent advances.)

- W. E. Spicer, A. Herrera-Gómez, SLAC-PUB-6303 and SLAC/SSRL-0042, August 1993.

- J.A. Nation et al., Proceedings of the IEEE 87, 865 (1999). (Comparison of a variety of cold cathode technologies, including field emitters and ferroelectric sources, for microwave tubes and $\mathrm{rf}$ accelerators.)

- K.L. Jensen, J. Vac. Sci. Technol. B21, 1528 (2003). (Review of emission theory.)

\section{Photoemission from Metals}

- $\quad$ E.M. Logothetis and P. L. Hartman, Phys. Rev. 187, 460 (1969).

- $\quad$ R. Yen, et al., Appl. Phys. Lett. 40, 185 (1982).

- H.E. Elsayed-Ali et al., Phys. Rev. B43, 4488 (1991).

- T. Tsang, Applied Physics Letters 63, 871 (1993).

- D.M. Riffe et al., J. Optical Society of America B10, 1424 (1993).

- B. Leblond, et al., Nucl. Instr. Meth. A372, 562 (1996).

- N.A. Papadogiannis, et al., J. Phys. D: Appl. Phys. 30, 2389 (1997); N.A. Papadogiannis, S.D. Moustaïzis, J. Phys. D: Appl. Phys. 34, 499 (2001). (Laser heating of electron gas and lattice and effects on photoemission.)

- K.L. Jensen et al., Appl. Phys. Lett 81, 3867 (2002). (Generalized emission model for field, thermal, and photoemission.)

\section{Metal Photocathodes}

- T. Srinivasan-Rao et al., J. Appl. Phys. 69, 3291 (1991).

- T. Srinivasan-Rao et al., Proc. of the 1997 Particle Accelerator Conference, Vancouver, 1997, IEEE, Piscataway, NJ, p. 2790. (Review of UV radiation can "cleaning" a metal surface and enhancing quantum efficiency for metal photocathodes.)

- X.J. Wang et al., Nucl. Instrum. Methods A356, 159 (1995).

- J.G. Neumann et al., Nucl. Instrum. Methods A507 498 (2003).

\section{High QE Alkali Antimonide and Alkali Telluride Photocathodes}

- P. Michelato, Nucl. Instrum. Methods A393, 455 (1997).

- A. di Bona et al., Nucl. Instrum. Methods A385, 385 (1997).

- S.H. Kong et al., J. Appl. Phys. 77, 6031 (1995). 
- D. Bisero et al., Appl. Phys. Lett. 69, 3641 (1996); ibid, 70, 1491 (1997).

- E. Chevallay et al., Proc of the XX Linac Conf., Monterey, CA., 2000, p. 110.

- A. Braem et al., Nucl. Instrum. Methods A502, 205 (2003).

- A. Breskin et al., Appl. Phys. Lett. 69, 1008 (1996). ( $\mathrm{H}_{2} 0$ \& $\mathrm{CO}_{2}$ poisoning)

- A. Aleksandrov et al., Phys. Rev. E51, 1449 (1995), A.V. Aleksandrov et al., Proc. of the 1996 European Particle Accelerator Conference, p. 1535. (GaAs response times less than $40 \mathrm{ps}$; Shorter duration pulses from III-V semiconductors use a thin active layer, though the $\mathrm{QE}$ is reduced because optical absorption is reduced.)

\section{NEA Photocathodes}

- J.S. Escher, "NEA Semiconductor Photoemitters," in Semiconductors and Semimetals, Willardson and Beer, eds., (New York: Academic Press, 1981), 15:195.

- R.L. Bell, Negative Electron Affinity Devices, (Clarendon Press, Oxford, 1973). (Note: temporal response calculated in Appendix A of this book is much slower than has been measured. Information on materials technology is outdated. However, underlying physics is well covered.)

- B.M. Dunham et al., Proc. of 1995 Particle Accelerator Conference, Dallas, TX, IEEE, Piscataway, NJ, p. 1030. (Measurement of the thermal emittance for NEA GaAs at room temperature as low as $35 \mathrm{meV}(406 \mathrm{~K})$.)

- S. Pastuszka et al., Appl. Phys. Lett. 71, 2967 (1997); S. Pastuszka et al., J. Appl. Phys. 88, 6788 (2000); D.A. Orlov et al., Appl. Phys. Lett. 78, 2721 (2001); V.V. Bakin et al., JETP Lett. 77, 167 (2003). (Cooling a NEA GaAs cathode to reduce thermal emittance.)

- T. Maruyama et al., Appl. Phys. Lett. 55, 1686 (1989). (Emission of polarized electrons from GaAs.)

\section{Photoemission from Metal Needle Cathodes}

- I. Boscolo et al., Proc. of the 1999 Particle Accelerator Conference, New York, 1999, IEEE, Piscataway, NJ, p. 1985. (Photoemission from ferroelectric cathodes.)

- C. Brau, Nucl. Instrum. Methods A393, 426 (1997).

- C. Brau, Nucl. Instrum. Methods A407, 1 (1998).

- C. Hernandez-Garcia, C. A. Brau, Nucl. Instrum. Methods A475, 559 (2001).

- C. Hernandez-Garcia, C. A. Brau, Nucl. Instrum. Methods A483, 273 (2002).

- K.L. Jensen et al., Nucl. Instrum. Methods A507, 238 (2003).

\section{Photoemission from Dispenser Cathodes}

- D.W. Feldman et al., Proc. 24th Int'l Free Electron Laser Conf., Argonne, IL 2003 (IEEE, Piscataway, NJ, 2002), p. II-15.

- K.L. Jensen et al., Appl. Phys. Lett. 83(6), 1269 (2003).

- K.L. Jensen et al., PRST-AB 6, 083501 (2003).

\section{Emittance Measurements and Issues}

- H. Tomizawa et al., Proc. of the 2002 European Particle Accelerator Conference, p. 1819; and J. Yang et al., J. Appl. Phys. 92, 1608 (2002). (Reductions in transverse emittance by improving spatial / temporal laser profile.) 
- F. Zhou et al., Phys. Rev. ST-AB 5, 094203 (2002). (Shows importance of cathode emission uniformity (involving uniform laser profile and QE) for minimizing transverse emittance.)

- S.G. Anderson et al., Phys. Rev. ST-AB 5, 014201 (2002). (Space charge and emittance measurement / theory discrepancies.)

- Ph. Piot et al., Proc. of the 2001 Particle Accelerator Conference, Chicago, 2001, (IEEE, Piscataway, NJ), p. 86. (Findings which contradict those of previous paper.)

- P.G. O’Shea, Phys. Rev. E57, 1081 (1998).

- M. Reiser, Theory and Design of Charged Particle Beams, (Wiley-Interscience, New York, 1994). (Canonical reference on beam dynamics and issues.)

\section{Emittance from Thermionic Cathodes}

- J. Haimson et al., Proc. of the 1997 Particle Accelerator Conference, Vancouver, 1997, (IEEE, Piscataway, NJ), p. 2808. (Thermionic emission from a conventional dispenser cathode controlled by high voltage pulsing on the gun giving 1 A with $3 \mu \mathrm{m}$ emittance.)

- T. Shintake et al., Proc. of the 24th ICFA Beam Dynamics Workshop on Future Light Sources, Hyogo, Japan, 2002. (Thermionic emission from a conventional dispenser cathode controlled by high voltage pulsing on the gun to give 3 A with $2 \mu \mathrm{m}$ emittance.)

\section{Ion Back Bombardment}

- J. Lewellen, Phys. Rev. ST-AB 5, 020101 (2002). (Concludes photocathodes in rf guns should not be susceptible to damage from ion backbombardment.)

- C.K. Sinclair, Proc. of the 1999 Particle Accelerator Conference, New York, (IEEE, Piscataway, NJ), p. 65. (Demonstration that photocathodes in DC electron guns show ion back bombardment damage.)

\section{Generation of Optical Beam Profiles}

- F.M. Dickey and S. C. Holswade, Laser Beam Shaping: Theory and Techniques, (Marcel Dekker, Inc., New York).

- F.M. Dickey and S. C. Holswade, Opt. Eng. 35, 3285 (1996).

- J.A. Hoffnagle and C. M. Jefferson, Appl. Opt. 39, 5488 (2000).

- M.A. Dugan et al., J. Opt. Soc. Am. B14, 2348 (1997).

- M. Weiner et al., J. Opt. Soc. Am. B5, 1563 (1988).

\subsection{Beam Dynamics of Photocathode rf Guns}

The brightness requirements for linac-based short wavelength FELs can nowadays only be fulfilled by photocathode rf guns. The development of rf guns began in 1985 by R. Sheffield et al. [1]. While in a DC gun the extraction voltage of $100-200 \mathrm{keV}$ is applied only over the small distance of the gap $(1-5 \mathrm{~mm})$ between the cathode and the anode, the cathode is mounted into an rf cavity in an rf gun. Thus the accelerating voltage extends over several centimeters and the extracted electrons are rapidly accelerated to some $\mathrm{MeV}$ beam energy. [Note that gradients of $\sim 40 \mathrm{MV} / \mathrm{m}$ can be achieved in a DC gun, which is comparable to typical values in rf guns working at $1.3 \mathrm{GHz}$ (L-band); however, given present $\mathrm{DC}$ voltage source limits of $\sim 500 \mathrm{kV}$, the beam energy from a DC gun is considerably lower than the beam energy from an rf photoinjector.] This fast acceleration leads to a reduction of space-charge-induced emittance 
growth. The cosine-square-like acceleration voltage that the electrons experience in a standing wave cavity may, on the other hand, lead to an rf-induced emittance growth due to the timedependent focusing of the rf field. The transverse rf-induced emittance growth scales with the bunch length and the transverse beam size, and can be kept small only by keeping the bunch dimensions small relative to the cavity dimensions and resonant frequency. Smaller bunch dimensions lead, however, to increased space-charge forces and detract from the beneficial effects of the fast acceleration to relativistic energies.

The beam dynamics in $\mathrm{rf}$ guns is thus driven by a complex interplay of space-charge forces, time-dependent rf forces, and static magnetic focusing forces from external solenoid magnets. The basic dynamics in the transverse and longitudinal planes are described in Ref. 2. Spacecharge forces play a critical role, since they depend not only on charge, size, and energy of the bunch, but also on details of its geometrical form. A uniform distribution in the transverse plane linearizes the space-charge forces in the limit of a long bunch and minimizes emittance growth due to nonlinearities. Since the transverse defocusing force depends still on the longitudinal position within the bunch, a correlated distortion of the phase space occurs, thus leading to a correlated emittance growth. This distortion can be corrected by manipulating the beam envelope downstream of the gun with focusing solenoid magnets [3]. A detailed theory of this emittance compensation scheme, concentrating on a special solution of the problem, has been worked out by L. Serafini and J. Rosenzweig [4]. The theory gives a guideline for a proper choice of strength and position of focusing and accelerating fields; the overall injector design relies, however, on simulations to incorporate higher-order effects.

An important step towards the realization of high-brightness beam injectors is the integration of higher harmonic cavities. These cavities allow decoupling of the transverse and longitudinal parameters of the injector, since longitudinal phase-space distortions can be corrected even if a very long bunch is launched. The basic concept of higher-harmonic cavities is not new; however, the full capabilities of this approach has been realized only recently [5].

Fast, semianalytical codes and slower, multiparticle tracking codes are available for the simulation of injectors. A comparison of codes can be found in Ref. 6 . The semianalytical codes make a number of simplifications but allow rapid scanning over a wide range of parameters. Tracking codes make fewer assumptions and allow a deeper insight into details of the phase space. They are also the starting point for start-to-end simulations of entire linear accelerators [7].

Benchmarking of these codes against experimental results is mandatory. Test facilities devoted to this task are running at various places in the world. As an example, Figure 2 shows the photoinjector test facility at DESY in Germany. Significant progress has been made in this field in recent years. Besides improvements in the beam diagnostics, which result in improved reliability of experimental results, it is of great importance to know the initial conditions for the simulation very precisely. This holds for the fields of cavities and solenoids but also includes a detailed knowledge of the laser parameters such as pulse length, form, transverse size, and internal structures like 'hot spots.' These input parameters for the simulation are difficult to obtain; however, a fairly good agreement of simulation and experiment is found only if all initial conditions are known and properly taken into account in the simulation [8]. 


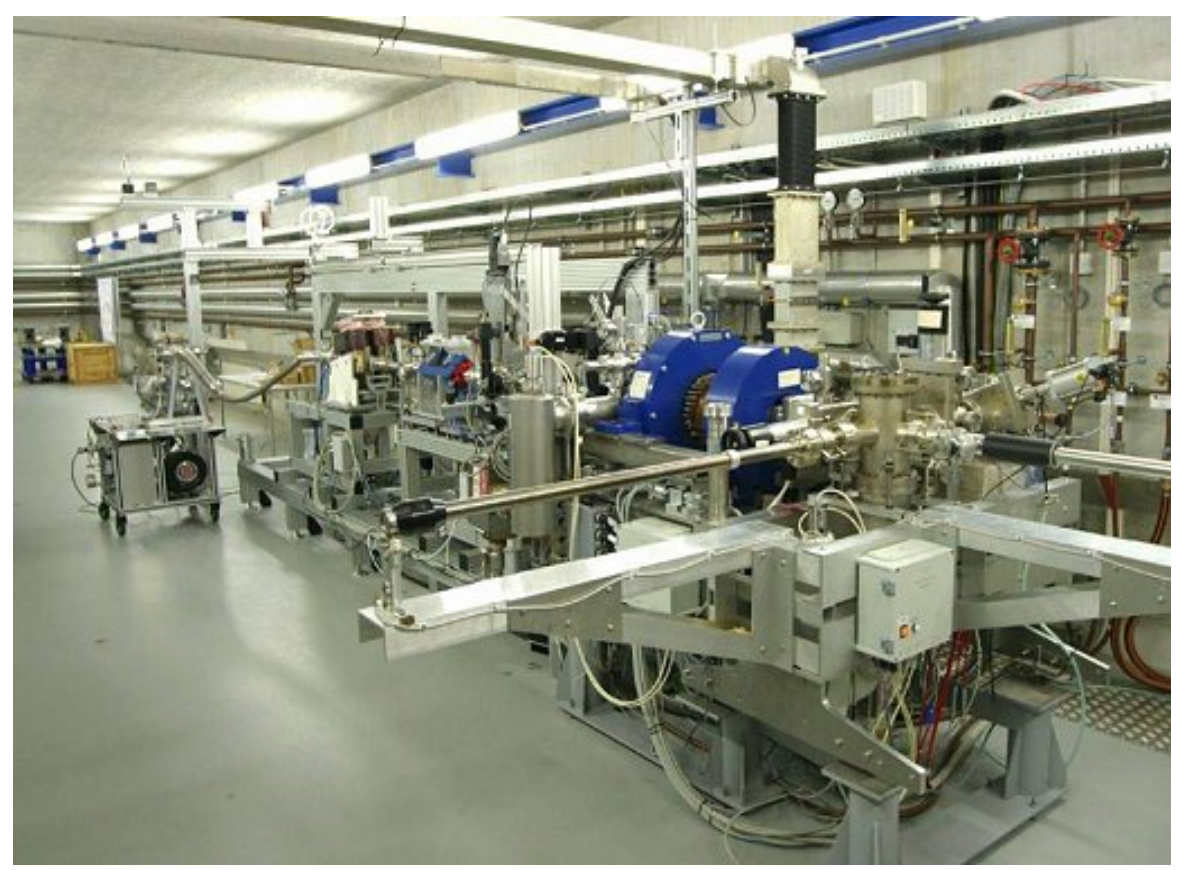

Figure 2: The photoinjector test facility at DESY in Germany.

Recent developments focus on the fine structure details of the electron beam, including particular study of the longitudinal phase space. It has been shown [9] that a microbunching instability can be driven by space-charge forces due to the magnetic bunching chicanes in linear accelerators. The origin of this instability points back to current fluctuations, which may occur due to imperfect laser pulses at the photocathode. Theoretical models and simulations are performed in parallel with encouraging first results. The dynamics of the first few centimeters inside the gun is particularly challenging from both the theoretical and the simulation points of view. A high resolution and a very large number of particles are required in the simulations in order to overcome numerical noise problems. Improvements in the codes are likely to be necessary in order to cope with these and future requirements.

While the general understanding of the beam dynamics in rf guns as well as theoretical and simulation tools seem to be adequate for injector designs as required by present-day projects such as the LCLS or the TESLA X-FEL, improvements are desirable and necessary to reach even smaller emittances. To realize the small emittances in practice requires improvements especially with respect to the control of the laser pulse parameters, i.e., pulse form and internal structure. The cathode will also become increasingly critical, both in terms of emission uniformity and intrinsic minimum obtainable emittance. A broader exploration of the available parameters space (e.g., bunch charge, bunch length at the injector) in conjunction with the overall machine layout and the FEL requirements has to be performed in order to find the optimal parameter setting. New schemes for the compensation or suppression of correlated space charge and rf-induced emittance growth need to be studied in simulations and eventually also experimentally. 


\section{References}

[1] J.S. Fraser et al., "High-brightness photoemitter development for electron accelerator injectors,” AIP Conf. Proc. No. 130, 598 (1985).

[2] K.-J. Kim, Nucl. Instrum. Methods A275, 201 (1989).

[3] B.E. Carlsten, Nucl. Instrum. Methods A285, 313-319 (1989).

[4] L. Serafini and J.B. Rosenzweig, PRE 55, 7565 (1997).

[5] P. Piot et al., "Generation of ultra-short electron bunches by cancellation of non-linear distortions in the longitudinal phase space," DESY-TESLA-FEL-01-06, 2001.

[6] C. Limborg et al., "Code Comparison for Simulations of Photo-Injectors," Particle Accelerator Conference 2003, to be published.

[7] ICFA Mini-Workshop on Start-to-End Simulations for X-ray FELs, http://www.desy.de/s2e/.

[8] C. Limborg et al., "PARMELA versus Measurements for GTF and DUVFEL," SLAC-Pub9556, 2002.

[9] E. Saldin et al., "Longitudinal Space Charge driven Micobunching Instability in TTF2 Linac,” DESY-TESLA-FEL-2003-02, May 2003.

\subsection{Beam Conditioning}

The performance of x-ray SASE FELs critically depends on the transverse beam emittance. The nominal normalized emittance for the LCLS is $1.2 \mathrm{~mm}$-mrad and for the TESLA X-ray FEL, 1.6 mm-mrad. In 1992, Sessler, Whittum, and Yu [1] introduced an idea that allowed considerable loosening of the requirements for small transverse emittance of FEL beams. The idea is to "condition'" a beam by introducing a correlated energy variation in the radial direction. Because the averaged longitudinal velocity of electrons depends both on energy and betatron amplitude, conditioning eliminates spread in the longitudinal velocity due to the emittance. In a conditioned beam, particles stay much longer in phase with the emitted electromagnetic field.

More precisely, conditioning changes a particle's energy in the beam by $m c^{2} \Delta \gamma$, where

$$
\Delta \gamma=\kappa_{u}\left(J_{x}+J_{y}\right) \text {, }
$$

where $J_{x}$ and $J_{y}$ are the actions for betatron oscillations in the $x$ and $y$ directions, respectively, normalized so that $\left\langle J_{x}\right\rangle=\left\langle J_{y}\right\rangle=\varepsilon_{N}$, where $\varepsilon_{N}$ is the normalized transverse emittance of the beam. The parameter $\kappa_{u}$ is determined by the properties of the undulator and parameters of the beam:

$$
\kappa_{u}=\frac{1}{2 \bar{\beta}} \frac{\lambda_{u}}{\lambda_{r}} .
$$

Here $\bar{\beta}$ is the averaged beta function in the undulator, $\lambda_{u}$ is the period of the undulator, and $\lambda_{r}$ is the radiation wavelength. Another useful parameter introduced in [2] is the dimensionless product of $\kappa_{u}$ and the bunch length in the undulator $\sigma_{z u}, a=\kappa_{u} \sigma_{z u}$.

For LCLS, with the nominal beta function $\beta=18 \mathrm{~m}, \kappa_{u}=5.8 \mu \mathrm{m}^{-1}$. For the nominal emittance 1.2 micron, Eq. (5) gives the scale of the necessary energy variation in the beam: $\Delta E=5 \mathrm{MeV}$. 
In practice, it might be more convenient to perform conditioning at the beginning of the linac, before the beam compression. It was pointed out in [2] that in this case the correlated energy spread required for conditioning is much smaller with the corresponding parameter $\kappa$ equal to $\kappa$ $=\kappa_{u} \sigma_{z u} / \sigma_{z}$, where $\sigma_{z}$ is the bunch length before compression. In the LCLS, the compression factor $\sigma_{z} / \sigma_{z u}=40\left(\sigma_{z}=1 \mathrm{~mm}, \sigma_{z u}=24 \mu \mathrm{m}\right)$ and conditioning before the compression requires $\Delta E=130 \mathrm{keV}$.

A study of FEL performance with conditioned beams was presented at the Theory Institute by A. Sessler. Numerical simulations show that for the parameters of future x-ray FELs, a conditioned beam with the nominal emittance has a shorter saturation length and allows a shorter undulator for the same output power. Alternatively, one can tolerate a larger transverse emittance beam with the same FEL power. It was pointed out that optimization of the undulator for a conditioned beam requires a stronger focusing.

Similar results were presented by S. Reiche [3] for the parameters of the TESLA XFEL. He also calculated the effects of overconditioning and underconditioning, and emphasized that gain length increases and the laser power decreases in both cases, although overconditioning has a slower roll off.

The crucial question for conditioning is how to generate a radial energy profile in the beam. In the original paper [1], they proposed using $\mathrm{TM}_{210}$ mode cavities immersed in a FODO lattice. Other methods were also considered including using a slow TM mode in a wiggler [4], and a laser with an undulator [5]. In Ref. [2] they proposed using solenoids with two rf cavities, which introduce longitudinal energy chirp in the beam (see also [6]). For the magnetic field in the range of several Tesla, such a conditioner can be relatively compact. It was found, however, that such a conditioner leads to an undesirable side effect - it increases the projected emittance of the beam by a factor of $a^{2}$, which is a very large number for XFELs. This growth of the emittance is caused by a focusing mismatch of the slices of the beam introduced by the solenoids. Presentation of a solenoid-based conditioner was given by G. Stupakov. The latest, though preliminary, results presented at the Theory Institute show that emittance growth can be considerably suppressed and almost eliminated in a system of many solenoids separated by quadrupoles with 90 degrees phase advance.

A. Wolski's talk presented several examples of conditioners that are matched and do not produce a projected emittance growth. Such conditioners can be described by the following Hamiltonian:

$$
H(J, \phi, z, \delta)=\frac{1}{L}(\mu+\kappa z) J,
$$

where $J$ and $\phi$ are the action and angle of the betatron oscillations, respectively, $z$ and $\delta$ are the longitudinal coordinate and the relative energy deviation, respectively, and $L$ is the conditioner length. The side effect of this conditioning is benign - it produces a phase rotation of slices of the beam, which depends on the longitudinal position within the bunch. Two new schemes presented by A. Wolski include 1) rf cavities in combination with a chromatic FODO beamline and 2) an $\mathrm{rf} \mathrm{TM}_{110}$ mode cavity with several sextupoles. The problem with these types of conditioners is that they are relatively weak - the largest value of the parameter $\kappa$ is on the order of $\sim 4 \times 10^{-4} \mu^{-1}$. Conditioning of an LCLS-type beam would require many passages through 
such a device. However, Wolski pointed out that such a conditioner can be inserted into a ring, saving the length of the conditioner in comparison with a linear system.

In addition to conventional approaches outlined above, A. Sessler proposed two conditioning schemes that use laser beams. In the first scheme, the Compton backscattering of laser photons off the beam is used to decelerate electrons. Due to radial variation of the laser radiation, the rate of deceleration depends on the radial position of the electron. Compton scattering is not a Hamiltonian process and should not cause emittance growth inherent to other techniques. A disadvantage of the method is that it requires a large number of scattering events, which is not easily achievable due to the small size of the Thompson scattering cross section.

In the second scheme, it was proposed to use a laser wakefield accelerator to condition an electron beam. A Langmuir wave excited by a laser pulse in a plasma has an accelerating field that varies in radial direction, and interaction of this wave with an electron beam introduces correlation between the energy and the radial coordinate. This approach requires smaller laser energy compared to the Compton backscattering method. However, a large radial electric field in the plasma wave may deteriorate the beam emittance.

At a dedicated session, a program of future research in the area of beam conditioning was discussed. Below we summarize some questions and the goals that were formulated at this session as necessary steps for a successful continuation of the beam conditioning research.

1. SASE FEL Theory and Simulations

- Study how overconditioning and underconditioning affect FEL performance.

- Consider possible increase of the energy spread during conditioning.

- Analyze effect of longitudinal variation of conditioning within the bunch.

- Analyze transverse coherence of FEL radiation of the conditioned beam.

- Study suppression of space-charge and CSR-induced instability due to correlated energy spread of a conditioned beam

2. Conditioning Techniques

- Continue work on conditioning techniques and identify the most promising candidate(s). Concept of a ring conditioner seems very promising since the amount of conditioning in one stage/cell is relatively small for most of the considered schemes.

- A better understanding of the effect of emittance growth during conditioning is required, which would incorporate the theory of matched solutions that do not exhibit the emittance growth.

- Are there any fundamental limitation of conditioning from Maxwell and Hamiltonian equations?

- Verify the effect of beam compression on conditioning.

- Eventually perform start-to-end simulation of an FEL with conditioner.

3. Experimental Studies

- Design a proof-of-principle experiment that would achieve beam conditioning.

- Design a conditioner ring. This includes a study and an optimal choice of beam energy, ring lattice, beam dynamics issues, etc. 
- Diagnostic of a conditioned conditioning. Consider the possibility of building a specialized energy analyzer that measures radial correlation of the beam energy.

\section{References}

[1] A. Sessler et al., Phys. Rev. Lett. 68, 309 (1992).

[2] P. Emma and G. Stupakov. Phys. Rev. ST Accel. Beams 6, 030701 (2003).

[3] S. Reiche, Nucl. Instrum. Methods A445, 90 (2000).

[4] P. Sprangle et al., Phys. Rev. Lett. 70, 2896 (1993).

[5] H. Liu and G.R. Neil, Phys. Rev. Lett. 70, 3557 (1993).

[6] N.A. Vinokurov, Nucl. Instrum. Methods A375, 264 (1996). 


\section{Outstanding Problems and Approaches for Their Solution}

In this section we discuss approaches to solving the issues that arise in developing highbrightness electron sources. The three areas of electron emission, $\mathrm{rf}$ gun beam dynamics and beam conditioning are each reviewed; beginning with general remarks followed by specific goals and the method for accomplishing them. Possible existing facilities capable of performing laboratory research are suggested, and each discussion ends with an assessment of the likelihood of success.

Implicit in the Institute's approach was the assumption that the path to a 0.1-micron, high-current beam to drive FELs in the future could be obtained from a low-thermal-emittance, highquantum-efficiency photocathode residing in an rf gun and beam conditioning transport. Other electron sources such as laser-driven plasmas [1] were not discussed. Future meetings of the Institute should include presentations on these and other advanced electron sources.

\subsection{Emission Process}

While there is no new fundamental physics related to the electron emission, there is a gap in our ability to combine all of the relevant phenomena into a complete and useful physical model. This gap needs to be closed since it now appears that the intrinsic cathode emittance will be the dominant quantity that limits our achieving beams of higher brightness from an rf gun. Therefore the outstanding problem for the emission process is to understand the details: How in detail are the photoelectrons emitted and what contributes to the thermal emittance and quantum efficiency $(\mathrm{QE})$ ?

The emission process can be separated into two parts: the cathode material 'intrinsic emittance' and the space-charge emittance. The Institute chose to use the term 'intrinsic' rather than 'thermal' emittance since it is observed that the zero charge emittance is larger than the expected thermal emittance due to several other effects unrelated to the bunch charge. In the effort described here, the emission process is assumed to concentrate upon the cathode material properties. The effects due to space charge are dealt with by inclusion of the new emission models into the beam dynamics codes, as described below.

\subsubsection{General Discussion of Goals}

The goal of the theoretical and experimental research would be to develop cathodes and techniques to achieve a total emittance of 0.1 micron with $1 \mathrm{nC}$ of charge. This appears possible but only if there are focused research efforts in the areas of electron emission, electron dynamics, and beam conditioning. The electron emission process presently used in many of the beam dynamics codes, like PARMELA [2] or ASTRA [3], simplifies the relevant physics. The emission effects are typically lumped into the initial particle distribution, in a way that the initial emittance fits to the thermal emittance. A simple model for the Schottky effect is implemented in ASTRA.

The theoretical collaboration should concentrate on developing the ability to compute the intrinsic emittance and QE from the cathode materials properties. Presently models to calculate the thermal emittance exist for the case of a purely thermal emission (see e.g., ref [4]) and for a 
monoenergetic, isotropic emission [5]. Both models are special cases and are not valid for the emission from all photocathode materials.

The second objective would be to integrate this understanding of the emission physics into a beam dynamics code like PARMELA or ASTRA. This can be achieved by forming a simulations code group within the theory collaboration.

It is also recognized that additional materials science data will be needed for the theory and simulations. Measurements will be required of the physical properties of the surface, especially the surface roughness, electrical, and photoelectrical properties. Near-field techniques could be used to map the spatial dependence of these data.

Once the emission models are integrated into the dynamics code, the emittance in a gun can be simulated and compared with experiment. In this regard, the Institute suggested the connection between theory and experiment be strengthened. Encouraging cooperation is essential to the successful implementation and understanding of new cathode materials and surfaces with low emittance and high QE.

\subsubsection{Summary of the Approaches for Emission Process Research and Development}

Goal: Develop the capability to compute the emittance and QE from first principles.

\section{Approach:}

- Form a theory and simulations collaboration with the goal of understanding the physics of emission and including it into a beam dynamics code. The possible institutions are University of Maryland, NRL, Argonne/APS, LANL, and DESY/TTF.

- Pick a limited number of cathode types which are in common use to study. These may be the metal cathodes $(\mathrm{Cu}$ and $\mathrm{Mg})$, dispenser-like cathodes, the PEA cathodes (CsTe, $\mathrm{KSbCs}$ ), and the NEA cathodes (Cs:GaAs). In choosing the cathodes to study, it is important to concentrate on the ones already in use or potentially usable at existing gun facilities. This allows direct comparison of the emission models with minimal hardware cost.

- Construct emission models that include the relevant physics for each cathode type. The model should begin with the cathode's physical properties and end with experimentally verifiable predictions of the emittance, $\mathrm{QE}$, etc. One result of this work will be to create a database of materials properties needed for the model.

- Research the properties needed for the materials database, both by literature searches and by measurements in the laboratory. The possible collaborators are Argonne/APS and NRL.

- Work with experimenters to design and perform experiments to verify the emission models. 
Goal: Integrate the emission models into the beam dynamics codes.

\section{Approach:}

- While developing the emission theory, begin the effort to incorporate the emission models into the beam dynamics codes. A major decision by the simulations collaboration will be whether to upgrade the existing codes or create new ones. The consensus of the Institute was that the present codes in use do not properly include the emission processes. However, it should be possible to build new low-energy 'front ends' for the existing codes that take over the computations once the beam energy is high enough. LANL, DESY/TTF, and SAIC are the possible working collaborators.

- Combine the simulations effort with the experiments to validate the simulations and keep the experiments relevant.

Goal: Develop a focused experimental program to verify the emission models and build facilities for sharing the new cathode technologies.

\section{Approach:}

- Form the Emissions Physics Experimental Collaboration, perhaps as part of a larger Gun Physics Experimental Collaboration. This group will concentrate on developing the facilities and experiments needed to support the Theory and Simulations Collaboration and to verify the emission models.

- The collaboration should agree on which facilities should specialize in what areas of research. For example, one facility could concentrate on the materials properties (ANL/APS), another on cathode fabrication (BNL), and another on low-energy emittance measurements (ANL/ITS, SLAC/GTF). The group should review the appropriateness and availability of existing facilities and their willingness to participate in the collaboration.

- Pool resources to design and build a load-lock system for the widely used S-band gun. This allows more labs to share the benefits of the new cathodes.

- Establish standards for the measurement of the thermal, slice, and projected emittances. Attempt to eliminate the existing controversy over emittance measurements.

- Consider establishing a single facility for cathode development. This could follow the European example, where a laboratory in Milan researches and fabricates cathodes for use at other labs. Since laboratories are expensive, it makes some sense to establish a single facility to make cathodes for everyone else.

\section{Probability of Success}

The success of the above-mentioned effort to produce new and better cathodes is good but difficult. It can be argued that since significant progress has already been achieved without any 
collaboration, then many more advances should be possible with the collaborative effort described above.

Even in the absence of significant improvements, at least we will have a better understanding of cathodes and the emission process. For example, presently we do not know if the cathode's intrinsic emittance can be decreased without compromising its QE. However, developing the capability to compute the intrinsic emittance and QE is certainly possible, and having the emission process 'front end' for the dynamics codes will tremendously improve electron gun simulations. The access of the gun community to new and better cathode facilities and capabilities would also greatly benefit the many gun facilities, both existing and proposed.

\subsection{Beam Dynamics of rf Photocathode Guns}

An outstanding problem of beam dynamics is that there have been only a handful of new gun concepts seriously investigated for ten or more years. The photocathode gun has been so successful that few have seen the need for anything else. And any the limitations of the photocathode gun, whether rf or DC, have always been attributed to either cathode or the drive laser limitations; that is, space-charge effects are mitigated by shaping the laser, and longitudinal space-charge effects result from laser fluctuations and cathode non-uniformities. In addition to space charge compensation [6], a concept now 14 years old, the only approach to improving beam quality was to build guns with as high a cathode field as possible [7]. This view is now changing. New rf cavity architectures that operate on higher-order modes [8] and can resonate at multiple frequencies have been proposed [9].

Achieving 0.1-micron emittance should be possible with the rf photocathode gun. Preliminary analysis of a two-frequency gun first proposed by Serafini and revisited by Dowell [9], gives 0.2micron emittance, but without inclusion of the intrinsic emittance. Therefore as stated in the emission process section, the intrinsic emittance is beginning to be the dominant contribution to the gun's emittance.

These results lead us to two major conclusions: 1) lower emittance from rf guns is possible; however, 2) the intrinsic emittance will dominate and require operation with long, pencil-like beams. Intrinsic emittance forces us to operate using a small cathode area, while space charge forces the bunch to be long. Long-bunch operation implies the gun should operate at DC [10] or low-frequency rf [11], to minimize accelerating field effects. However DC and low-frequency guns are limited to achievable peak fields of less than $30 \mathrm{MV} / \mathrm{m}$ [12]. On the other hand, the two-frequency simulations indicate that rf fields of $100 \mathrm{MV} / \mathrm{m}$ can be made more linear, or DClike, with the addition of harmonics [9]. Pulsed diodes have been investigated but have very small integrated acceleration lengths, i.e., similar to the DC gun; the gun energy is low.

Goal: Investigate new gun designs capable of producing 0.1 -micron emittance 1-nC beams.

\section{Approach:}

- Create a gun collaboration with members from the various rf gun facilities as well as representatives from the novel (plasma, laser-accelerator, etc.) gun communities. 
- Perform a survey of all the electron source research facilities in the world. Compile a complete listing of each facility's capabilities and willingness to participate in the collaboration. Align capabilities with the experimental plan.

- Work with the Theory and Simulations Collaboration on experiments to understand the physics of bright beams and to validate theories and simulations.

- Determine which two or three concepts are the most promising and develop them in parallel. Consider approaches consistent with existing cathodes or those being studied by the Emission Physics Experimental Collaboration. Don't assume a 'miracle' cathode or 'wonder' laser will be invented.

- Generate realistic mechanical and electrical designs to study which concepts are practical to build.

- Fabricate one or two designs and test them at the appropriate facility. A promising approach is a two-frequency gun built to operate at L-band $(1300 \mathrm{MHz})$ and $3900 \mathrm{MHz}$, and tested at the FNPL/FNAL facility. The advantage of performing this test at FNPL/FNAL is that Fermi Laboratory already has a photocathode gun facility with cathode and drive laser capabilities, as well as rf sources at the required fundamental and third harmonics. The 3.9-GHz source is being developed for a longitudinal phase-space linearizer in collaboration with DESY/TTF. A second possible facility would be SLAC where the S-band version could be built and tested. However, SLAC has available only the fourth harmonic of the $2856 \mathrm{MHz}$, rather than the required third harmonic, and developing a new third harmonic klystron maybe too expensive for the R\&D project.

\section{Probability of Success}

Successfully understanding beam dynamics in an $\mathrm{rf}$ gun well enough to achieve 0.1 microns will be difficult but possible. The inclusion of photoemission physics in the simulation codes is essential as the intrinsic emittance will define the beam dimensions in the gun. And our extensive knowledge of rf fields and our ability to custom shape them should allow successful production of this low-emittance beam. However, since space-charge forces will demand a very long bunch from the gun, preserving this beam quality during compression and transport to the undulator will become a new challenge. Therefore, further theoretical and experimental work is needed in these areas as well. Currently we do not know if such a bright beam can be preserved, given the usual manipulations performed between gun and undulator.

\subsection{Beam Conditioning}

Beam conditioning has the potential to improve FEL performance with poor quality electron beams. There has been significant work in just the past year, and the problem has progressed to the study of the limitations and consequences of the various concepts. The status of beam conditioning and goals for successful implementation of this interesting concept is summarized in Section 2.3. The comments below are in addition to those given there.

A. Wolski (LBNL) described schemes where the conditioning is weak and would therefore require either a very long beamline or multiple passes through the same device. It appears a ring beamline or accelerator is preferred since the beam will need to pass through a single conditioner thousands of times. However, proposing a ring to condition low-emittance beams necessitates the inclusion of many other phenomena, such as coherent synchrotron radiation, which will 
destroy the beam quality. The ring idea is a good one, but requires considerably more theoretical work.

In addition, what is lacking is an experimental facility for testing the more promising ideas. A possible facility is the Particle Accumulator Ring (PAR) at ANL/APS. There is a good chance that the PAR will become available not only because positrons are not used in the APS, but also because of the APS effort to directly inject high charge bunches. Serious thought should be given to its possible use as a ring for testing beam conditioning and related concepts.

\section{Probability of Success}

Success at beam conditioning would have a tremendous impact on short-wavelength FELs. Even with improved beam quality from the gun, beam condition would have great benefits. However, the most promising approaches are weak and require long beamlines or rings. Thus careful design of the beam transport, whether it's long or a ring, will be needed to preserve the conditioning. This is a problem for the experimental demonstration. While using an existing ring, like the PAR, is attractive, in all likelihood it does not have the needed optical properties. Therefore, even after the conditioning approach is chosen, there is still considerable effort involved to design the appropriate ring.

Inclusion of a weak beam conditioner will also have a major impact on the overall design of the FEL. If a ring is used immediately in front of the undulator, the bright beam will strongly radiate in the arcs, deteriorating its quality. If the conditioning is done at low peak current, then preserving the conditioning in a compressor becomes an issue. And if the conditioning ring includes bunch compression, there is the additional complication of emittance preservation in the presence of the CSR [13] and longitudinal space-charge instabilities [14].

The tremendous benefits of beam condition make it essential that we continue the theoretical studies. However, more theoretical work is required before constructing a conditioner.

\section{References}

[1] A. W. Chao et al., "Space charge dynamics of bright electron beams," PRST-AB 6, 024201(2003), and references therein.

[2] L. M. Young, "PARMELA," Los Alamos National Laboratory Report LA-UR-96-1835 (Revised July 17, 2003).

[3] K. Flöttmann, ASTRA User Manual, http://www.desy.de/ mpyflo/Astra_dokumentation/.

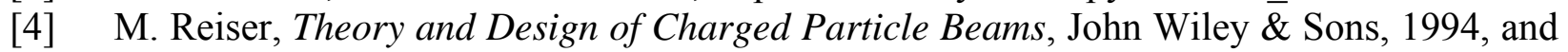 J.D. Lawson, The Physics of Charged Particle Beams, Oxford Science Publications, pp. 209-214.

[5] K. Flöttmann, "Note on the Thermal Emittance of Electrons emitted by Cesium Telluride Photo Cathodes," TESLA-FEL 97-01, 1997; see http://tesla.desy.de/new_pages/FEL/ FELnot97.html.

[6] B.E. Carlsten, Nucl. Instrum. Methods A285, 313-319 (1989).

[7] S. Troz et al., "High Power Operation of a $17 \mathrm{GHz}$ Photocathode RF Gun," AIP Conference Proceedings 398, pp. 717-729 (1997).

[8] J. Lewellen, PRST-AB 4, 040101 (2001). 
[9] D.H. Dowell et al., "A Two-Frequency RF Photocathode Gun," FEL 2003 Conference Proceedings (to be published).

[10] Institute presentation by H. Bluem, AES; see http://www.aps.anl.gov/asd/theory/talks/ Thur-Bluem.pdf

[11] J.-G. Marmouget et al., Proc. of EPAC2002, pp. 1795-1797 (2002).

[12] T. Siggins et al., Nucl. Instrum. Methods A475, 549-553 (2001).

[13] E.L. Saldin et al., Nucl. Instrum. Methods A398, 373-394 (1997).

[14] E.L. Saldin et al., "Klystron Instability of a Relativistic Electron Beam in a Bunch Compressor," Deutsches Elektronen-Synchrotron Report No. TESLA-FEL 2002-02, 2002.

\subsection{Simulation Tools}

\subsubsection{Introduction}

There is a need for a research and development program to develop modeling and simulation tools to support the design and development of high-brightness sources. This stems from the need for high-brightness, high-average-current beams for FELs. Currently, rf photocathode guns are the prime candidates for such FEL sources.

The currently available set of simulation codes fails to model the emission physics well and is not adequate to model the next-generation rf photocathode guns. For these next-generation sources, new simulation tools will be required for theoretical research and design.

\section{Findings}

The community at large does not have a complete set of simulation tools to support their needs. The inadequacy in modeling tools ranges from single-component modeling tools-like those needed just for cathode modeling - to the full system end-to-end modeling tools. Many issues need addressing, including the following:

- Data format and data-type file format differences between simulation codes.

- The need for open source software to support scientists' changing needs.

- The need for long-term core code maintenance.

- The need for new tools that meet our current needs.

The above list has been identified as requirements that are necessary to minimize the limitations in current simulation capabilities of the community. Other scientific communities have grown to develop simulation capabilities that provide the researcher with necessary tools, and this list has captured the essence of what similar approaches will help this community. New tool suites have been very effective at giving the researcher a predictive capability that often allows firstpass design success for components in many cases. They are also greatly effective at helping the theorist gain intuition that helps direct the design process more effectively.

Existing codes used for rf guns do not support four important needs. They are the following:

1. The need for an effective photoemission model. 
2. The need to model the detailed interbeam particle behavior, including important image charge effects.

3. Fine structure and beam-edge details.

4. Large-scale parallel capability.

The lack of physics-based photoemission models in currently available codes, as mentioned in 1. above, ultimately limits the simulation effectiveness. Current models simply insert charged particles into the simulation, but have no basis for the process except that they follow some prechosen time/spatial profile that has no self-consistent means for its phase-space distribution. Current scientists just use these models as is and hope that the beams produced show some semblance to reality. In fact, the community has identified that they don't.

Interbeam particle modeling in 2 . is very important, as the current trend is to lower beam emittance. Lower-emittance beams have been identified as having a dominant role in the significant reduction of machine size and cost. Current finite-difference codes struggle to accurately model low-emittance beams when these beams exist in large cavities, as found in $\mathrm{rf}$ cathodes. This is due to the difference in spatial scales between the beam and the cavity, and the meshing limitations of finite-difference codes. Other popular codes don't include the image charges of the beam bunch, which will affect the particle dynamics as the beam leaves the cathode. Codes that better deal with disparate spatial scales will provide a solution to these limitations.

The issue of component geometrical structure and beam-edge details mentioned in 3 . affects both current and future designs. The beam bunch that needs to be modeled has a spatial extent requiring mesh resolution that is simply not met in current models. To capture the interdynamics of the particles in a beam of limited axial and transverse extent requires a sufficiently fine mesh in all dimensions, but only within the needed regions. To capture fine details of its behavior, the beam edge requires a higher mesh density than the internal portions. Current codes simply don't capture this, and adaptive or conformal mesh codes will provide big advantages. Additionally, gun designs that include multiple beam systems or pin-type emitters simply cannot capture the fine scale of the fields, emission, and particle dynamics in the vicinity of the emitter edges without improved meshing capabilities. Conformal meshing is key to achieving this.

Lastly, the need for at least an order of magnitude increase in the particle count used to model the beamlet is important to properly populate the particle distribution in order to capture the important head and tail dynamics that the rf gun developer must include to design these beam sources. Greatly increased particle counts are also needed for improved statistics and are necessary for modeling reduced-emittance beams that are envisioned. Although some of the simpler numerical models may approach one simulated particle per real particle, the fully 3D models do not currently come close to this limit.

\subsubsection{Development Plan}

In this section, research and development plan topic areas are presented that address the modeling and simulation needs of the community. 


\section{Basic Requirements}

- A design tool to enable rf photocathode source technology for high-brightness beams

- Design tool should be based on the latest EM PIC modeling methods, which include:

- Conformal meshes, nested meshes

- Parallel computing

- Physics theory combined with empirical data needed to provide new photocathode emission models

- Improved user-controlled particle distributions

- User Environment with a graphical user interface developed with community input, including:

- Intercode data file manipulation

- An input wizard and user help

- Predeveloped examples for tutorials and usage review

- Job submission and job queuing

- Pre- and postprocessing

- Run time information and diagnostics for both status and appropriate physics

- Ability to do simple models mode for single-PC runs vs. workstation mode for more elaborate calculations

Essential physics and theory that must be captured by the simulation tools:

- Detailed photoemission process

- Beam-bunch microinstabilities, longitudinal space-charge instabilities

- Low emittance beams to be evolved accurately

- Beam head/tail effects

- Self-field images from the cavity/injector surfaces

- Resolving beam, field, and edge effects (e.g., in pin-type cathodes)

New design tool must include:

- Both frequency domain (for cavity modes) and time domain (for PIC)

- Sophisticated emission model to capture photoemission process

- An efficient conformal mesh to model:

- Emission region and large aspect ratio between beam and cavity

- Gross and fine geometric features and enhanced beam-region mesh

- Hybrid structured/unstructured mesh

- Finite Element (FE) approach for accuracy (with FD option for speed)

- Parallel architecture

- For accuracy, large problem size, speed, etc.

Emission model development considerations:

- Theoretical emission models need to be developed

- Emission models must be made compatible with simulation codes

- Experimental validation must be made 
- Conditions at the cathode are critical-

- What combination of optimal emission area, bunch duration, and fields (static or dynamic) needs to be quantified?

- Issues for insertion into simulation codes:

- Capture theoretically and empirically based emission model into code

- Code must handle the small beam bunch in a large cavity, accounting for interbunch, self and image effects

- Capture theoretically and empirically based models for surface roughness and machining effects

\section{Development Roadmap}

The currently available set of simulation codes fails to model the emission physics well, and is not adequate to model the next-generation rf photocathode guns.

A Modeling and Simulation development roadmap to mitigate this inadequacy is identified to be as follows:

- Photocathode electron emission/injection is currently not well treated

- New models are needed

- Based on experimental measurements and theory

- There is often a large aspect ratio between the rf cavity as compared with the beam size

- Dealing with disparate spatial scales can be important

- Must capture beam-bunch detailed distribution evolution

- Longitudinal space-charge instabilities and microbunching are believed to have a dominant role in the early-time evolution of the beam distribution

- There is a need to track large number of particles within a small bunch

- This requires fine meshing in the vicinity of the beam, possibly with a moving window or sub-mesh

- This requires the ability to efficiently handle large numbers of particles

- Parallel code architecture from the outset

- 2D and 3D modeling capability

- 2D: gross (axisymmetric ) effects captured - rapid progress and intuition

- 3D: nonaxisymmetric effects are important

- off-axis effect of the drive window and the laser source, the resultant noncircular beam, as well as downstream focusing from nonaxisymmetric/nonaligned magnetic fields and beamline components

- Meshes: nonconformal (for speed) and conformal (for precision)

- Possibility of a numerically-induced instability growth from nonorthogonal meshes.

- Code should be fashioned to handle Cartesian meshes - nonconformal

- As an unstructured domain - nonlogical Cartesian mesh

- As a structured domain (logical mesh), using masking of the geometry

- Both with stairstep corrections

- Evolutionary development plan — with planned improvements

- Level 1 - conformal structured/unstructured hexahedral mesh

- Level 2 - conformal unstructured tetrahedral mesh

- Level 3 - conformal hybrid structured and unstructured 


\section{Resources Required}

\subsection{Background}

It is proposed that research in high-brightness beams be patterned on research done at national accelerator facilities by scientists of every discipline:

- Costly technical infrastructure for accelerator R\&D should be constructed, maintained, and operated as user facilities at national laboratories.

- This infrastructure would be available for research on a competitive peer-reviewed basis, as is the case for users of neutron sources or synchrotron sources.

It would be a natural extension of the national labs' missions to provide a few such facilities for use by university researchers. Facilities that could be used for general accelerator-based R\&D are constructed as part of larger accelerator complexes (high-energy and nuclear physics accelerators, synchrotron sources, neutron sources, etc.), generally without provision for their use to support more speculative accelerator research. This unfortunate situation should be corrected; accelerator research at universities can and should be encouraged through competitive grants. Expensive infrastructure at national laboratories can and should be made available to university researchers in analogy with light sources and neutron sources.

\subsection{Areas of Opportunity}

The following is an attempt to subdivide the challenges identified at this Institute meeting into four categories, each of which can constitute an independent research effort.

\subsubsection{Properties of Cathode Materials and the Determinants of Intrinsic Emittance}

- Current-independent effects

- Current-limiting effects

- Effect of bulk and surface materials properties on gradient at the cathode

This category is intended to place emphasis on the structural and chemical properties of materials and surfaces as they are made to produce free electrons by photo- or thermionic emission. Research in this area requires theoretical/numerical studies and facilities for the preparation and characterization of cathode materials and surfaces, lasers for photoemissivity studies, and highvoltage sources and test stands for in-vacuum measurements of cathode emissivity combined with instruments to study adsorbed species on the cathode surface under realistic operating conditions. These facilities may be found at national labs and many universities around the country. Relevant research disciplines include surface chemistry and physics, nanoscale structures, and solid-state physics.

Perhaps two or three small research groups (principal investigator + postdoctoral fellow + graduate student) working for 5-10 years could have considerable impact on accelerator science. 


\subsubsection{Early Stages of Acceleration (Guns and Space-Charge Effects in Acceleration)}

- Effects of gun design on gradient and focusing at the cathode

- Compensation of transverse space-charge forces

- Compensation of longitudinal space-charge effects

The second category is intended to place emphasis on issues of gun design and space-chargedominated beam transport. Research in this area requires access to high-voltage DC sources and/or rf sources, facilities for accelerating beams to $5-50 \mathrm{MeV}$, and a suite of diagnostics for the accelerated beam. Facilities of this type may be found at universities, but are more commonly found at national laboratories. Perhaps an ideal facility would have rf power sources with outputs of 5-50 MW at several frequencies (500 MHz, 1.3 GHz, $2.9 \mathrm{GHz}, 5 \mathrm{GHz}, 12 \mathrm{GHz}$, etc.). DC and pulsed-power sources might be required for some experiments, as well as high-power CW rf sources and high-power lasers. Clearly the price of admission to this kind of research can become rather high. However, the tools design and construction of normal-conducting electron guns (design software, light machine shop) are well within the reach of many universities. At this time there are dozens of interesting gun designs published in the literature; the rate at which these guns are constructed and tested is very low by comparison.

An optimistic estimate of the scale of effort for this category might be made by imagining that there are about 20-30 gun designs suitable for testing over the next ten years. A prototype gun can take 1-2 years to design and construct, and 3-6 months to test. A single university-based research effort ( $\sim 3$ persons) might produce 3-4 completely distinct gun designs in ten years, so perhaps 4-6 groups and two "all-purpose" gun test facilities would support this level of effort. Of course, it is difficult to anticipate what an "all-purpose" gun test facility might include. It might be more economical to create a larger number of less versatile test stands where essential and expensive infrastructure already exists.

\subsubsection{Bunch Compression}

- Limiting degradation from CSR

- Limits of chirping for magnetic bunch compression

- Nonmagnetic (velocity) compression

This third category emphasizes optics and current- or quality-limiting phenomena for "relativistic" beams (for which space charge effects are usually ignored; however, this border is fuzzy and depends on both energy and current density). Theoretical and computational studies of bunch compression require specialized software. Indeed, software development for such computations is itself an interesting area of research. Useful simulations can be done with very modest computers. At this time, theoretical and numerical studies of bunch compression are extremely active areas of research, motivated by efforts to design free-electron lasers and linear colliders. The problem of bunch compression without undue degradation of emittance is of fundamental importance to the design of free-electron laser facilities. Theoretical studies of emittance degradation and the development of compression schemes that minimize degradation should be encouraged accordingly.

Experimental tests of bunch compression require very bright electron beams ranging in energy from $5 \mathrm{MeV}$ to $250 \mathrm{MeV}$; compression at $2-5 \mathrm{GeV}$ is of great importance to hard $\mathrm{x}$-ray free- 
electron lasers such as TESLA and LCLS. At all energies, detailed experimental studies are at a very preliminary stage. There are a few laboratories and universities that can provide access to 200-600 MeV electron beams. Only a few labs in the world have higher energy electron linacs. To date, linacs with bunch compressors are not readily available for research because they are dedicated injectors for other accelerators or else part of free-electron laser experiments. Even if a suitable linac is available, construction of a novel bunch compressor design for experimental purposes is a costly activity. Cost notwithstanding, more attention will surely be devoted to experimental studies of bunch compression in the next few years, primarily at national labs. University-based researchers could make valuable contributions to experiments in focused areas such as diagnostics.

\subsubsection{Other Advanced Techniques}

- Beam conditioning

- Emittance exchange

- Properties of beams in femtosecond and attosecond time scales

The fourth category is admittedly a catch-all for novel ways to produce electron beams enabling extraordinary synchrotron sources. Activities in this category are largely biased toward theoretical/computational studies; attempts to produce and characterize "flat" electron beams comprise a notable experimental effort in this category. Some intriguing ideas have been developed in the past few years. Since this category includes the most speculative research, it is reasonable to expect that the best way to encourage progress is to see that research in the first three categories is properly supported; breakthrough concepts and "surprises" will follow in due time.

\subsection{Longer-Term Commitment and Education}

This assessment is intended to provide a qualitative or, at best, a rough quantitative estimate of the appropriate scale of effort that might be reasonable for each category; the authors do not mean to imply that they have dismissed the existence or quality of existing accelerator research user facilities or past and ongoing efforts in these areas. The intent of this assessment is to argue that these categories should be addressed within stable research communities on a longer-term basis. The activities of these communities can and should support the needs of specific accelerator projects; however, a properly supported community should also include research activities that are not dictated by the schedule or performance goals of a specific project.

The word community is used here to refer to a collection of researchers and groups that

- Compete with each other for research funds, and

- Educate students who can pursue career options within the community.

\subsection{Costs}

Only cost estimates for "generic" facilities and research groups will be suggested here. The greatest uncertainties are associated with national laboratory user facilities, which may be able to reduce initial costs by using existing infrastructure. 
The setup of a national laboratory user facility would have an initial cost of $\$ 500 \mathrm{~K}-\$ 2,000 \mathrm{~K}$, a staffing level of 3-6 FTEs, and an annual budget for materials and equipment of about $\$ 500 \mathrm{~K}$ $\$ 1,000 \mathrm{~K}$. Three or four "average" size facilities would cost about $\$ 5 \mathrm{M}$ initially and $\$ 4 \mathrm{M}$ per year. These estimates assume that the facilities are co-located with national user facilities that already have trained staff and specialized support infrastructure that can be shared.

A university-based research effort (funding $1 / 10$ of a faculty principal investigator, one graduate student, and one postdoctoral fellow) would cost about $\$ 200 \mathrm{~K}-\$ 350 \mathrm{~K}$ per year; the initial equipment investment required to start a university-based research group is estimated to be $\$ 400 \mathrm{~K}-\$ 800 \mathrm{~K}$, perhaps spread over four years. The cost of operating ten such groups would therefore be in the range of $\$ 2 \mathrm{M}-\$ 3.5 \mathrm{M}$ per year, with startup costs of the order of $\$ 4 \mathrm{M}-\$ 8 \mathrm{M}$, spread over several years.

An effort on this scale (ten groups) would produce about two Ph.D.s per year, still many fewer than needed in the accelerator science and technology disciplines. A Ph.D. production rate of $\sim 40$ /year would approximately match national demand; this is significantly more than the production rate today.

The expense estimates above do not include the cost of operating a larger-scale accelerator. Some excellent accelerator facilities for beam physics research may be found at several universities. Generally their operating costs are low, but they are not operated as "national" user facilities.

Summing user facilities and users, the annual cost would be $\$ 8 \mathrm{M}-\$ 12 \mathrm{M}$ per year, with one-time startup costs in the range of $\$ 9 \mathrm{M}-\$ 13 \mathrm{M}$. Though this paper addresses some specific areas of research related to high-brightness beams, the envisioned user facilities could support a wider range of research efforts, relevant to accelerators for nuclear and high-energy physics. 


\section{Acknowledgements}

We thank the participants of the ANL Theory Institute on Production of Bright Electron Beams for their presentations and for their lively contributions during the discussion sessions. The list of participants can be found in Appendix $\mathrm{C}$ of this white paper.

We also thank Z.R. Huang for his calculations of the FEL performances for various beam parameters and C. Sinclair for discussions on the emission process and his help in compiling references. 


\section{Appendix A - Present Facilities}

These descriptions of facilities and capabilities for electron gun research were filed by the participants of the ANL Theory Institute on Production of Bright Electron Beams. The list is therefore not comprehensive.

\section{AES/JLab (Hans Bluem, AES)}

Jefferson Laboratory operates a multikilowatt, average power, sub-picosecond free-electron laser covering the mid-infrared spectral region, that has recorded the highest FEL power levels achieved in the IR regime. Extensions to $250 \mathrm{~nm}$ in the UV are under construction. The program and this new user facility derives from the primary mission of Jefferson Laboratory, namely, nuclear physics research and the world's first large superconducting accelerator for generating continuous multibillion-volt beams of electrons, called CEBAF. The new FEL upgrade will enable operation from 0.25 microns in the ultraviolet to 14 microns in the mid-infrared and with average powers up to 10,000 watts and rapid tunability. The electron beam power in the device is $1.5 \mathrm{MW} \mathrm{CW}$.

Jefferson Laboratory is also in the process of setting up an Injector Test Stand (ITS) with about 1 MW of 748.5- MHz rf power, ample cryogens for superconducting accelerator tests, and all other required infrastructure. The purpose of this ITS is to develop high-average current injector concepts. Advanced Energy Systems (AES) is almost finished with the fabrication of a 3-cell cryomodule for use with an existing DC gun that will deliver $100 \mathrm{~mA} \mathrm{CW}$. This device will be tested in the ITS. Two other AES projects will deliver other injector components for testing in the ITS. The first is an injector cryomodule with a third-harmonic cavity to linearize the longitudinal phase space and the other is a half-cell fully superconducting rf gun similar in style to that of FZ-Rossendorf.

\section{ANL/ITS and AWA (John Lewellen and Wei Gai, ANL)}

\section{Injector Test Stand (ITS):}

The Advanced Photon Source (APS) Injector Test Stand (ITS) is a small multi-role facility located adjacent to the APS injector linac and drive laser room. Intended primarily as a highbrightness photoinjector test bed, the ITS is also used to validate APS linac thermionic-cathode rf gun performance, and can also be used for operator training purposes.

The ITS is unique in that although it is powered by a single 35-MW klystron, three independent high-power rf feeds are available within the enclosure. Two high-power variable power dividers, two high-power phase shifters, and three circulators allow the power from the klystron to be divided arbitrarily between the ports, with an arbitrary phase relationship. Port 1 is capable of accepting the full power from the klystron, Port 2 is limited to $10 \mathrm{MW}$, and Port 3 to $5 \mathrm{MW}$ due to circulator power limits.

The ITS beamline is constructed using a 1-m by 3-m optical table as a "substrate," with a modular breadboard and rail system providing the actual beamline supports. This allows great flexibility in terms of reconfiguring the beamline for new experiments and results in considerable 
savings in terms of both time and materials. Quadrupoles and steering correctors are connected to their power supplies by quick-disconnect multi-pin connectors. These both greatly speed the process of removing or installing a magnet and guarantee that all connections (power, temperature sense, etc.) are made up properly.

An EPICS control system is used for the ITS; this allows the ITS to capitalize on the large hardware and software investment in EPICS already made at the APS. In particular, the entire suite of data collection, processing, controls, and feedback software can run without modification on the ITS. This is useful not only from an experimental viewpoint, but also from the perspective of operator training.

Presently, a ballistic bunch compression gun is installed in the ITS. The beamline is configured for energy-spread measurements and includes a combination zero-dispersion dogleg and spectrometer branch line. Future plans include $\mathrm{THz}$ radiation generation tests, long-pulse drive laser tests with an APS injector gun, construction of a secondary beamline for operator training and repair validation purposes, a DC/RF hybrid gun test in collaboration with DULY research, and the installation and test of a higher-order mode-photoinjector.

\section{Argonne Wakefield Accelerator (AWA):}

The advanced accelerator program at the Argonne High Energy Physics Division is focused on advancing the physics and technology of beams, particularly new approaches to beam acceleration and instrumentation important to the world high-energy physics program, such as the development of new techniques for accelerating electron beams to high energies. The present focus is on accelerator research in areas that require intense, short-pulse electron beams; dielectric-based wakefield acceleration in structures; plasma wakefield acceleration and focusing; generation of high-power rf using dielectric-lined waveguides and other types of slowwave structures; and enabling technologies of photocathode-based electron sources necessary to produce electron beams with the required characteristics for the all of the above.

We operate an experimental facility, the Argonne Wakefield Accelerator (AWA), that has the following major components: a novel high-current, photocathode-based electron source; a linac to produce a $15-\mathrm{MeV}$ drive beam; a $4-\mathrm{MeV}$ photocathode gun used to produce the witness beam; a UV laser system with sufficient energy per pulse to produce electrons from both sources; beamlines to transport both beams through the wakefield device under test; and rf control and data acquisition, shielding, safety systems, etc. Also a gun test stand is available for high-current beam or high-brightness beam characterizations from new photoinjectors. Currently a new AWA gun can produce beam of 1-100 $\mathrm{nC}$ with rms bunch length of 2-5 ps at $7 \mathrm{MeV}$. The calculated emittance is on the order of $2 \mathrm{~mm}-\mathrm{mrad} / \mathrm{nC}$ for high charges (10-100 nC) and $1 \mathrm{~mm}-$ $\mathrm{mrad} / \mathrm{nC}$ for low charges $(0.5-2 \mathrm{nC})$.

\section{BNL/DUV-FEL/ATF (Xijie Wang and Jim Murphy, BNL)}

The Brookhaven National Laboratory (BNL) DUV-FEL is a dedicated platform for FEL and other linac-based light source technologies R\&D. The main focus at the DUV-FEL is to develop and explore the laser seeded HGHG FEL. We also carry out R\&D in electron beam generation, compression, and ultrafast beam instrumentation at the DUV-FEL. 
The DUV-FEL is a laser linac facility based on a high-brightness photocathode rf gun injection system. The DUV-FEL facility consists of a 1.6-cell BNL GUN IV, four sections of 3-meterlong SLAC-type linac, a four-magnet chicane bunch compressor, an rf-synchronized Ti:sapphire laser system, and HGHG undulators. The picoseconds electron beam produced by the rf gun is quickly accelerated to about $70 \mathrm{MeV}$ by two sections of the linac. The electron beam is compressed down to sub-ps by the chicane before it is further accelerated by the last two sections of the linac. The maximum electron beam energy that can be reached at the DUV-FEL is about $200 \mathrm{MeV}$. The Ti:sapphire laser system is capable of producing $150 \mathrm{fs}$ to several picoseconds long laser pulses with about $50 \mathrm{~mJ}$ output. It is used both to drive the photocathode $\mathrm{rf}$ gun and to seed the HGHG FEL. One of the unique features of the DUV-FEL laser system is that one single laser system is capable of producing two rf-synchronized laser pulses with both pulse length and relative delay adjustable by employing two grating laser pulse compressors. Both the linac and laser system are capable of operating at $10 \mathrm{~Hz}$.

The following topics related to the electron beam production, preservation, and diagnostics will be actively pursuit at the DUV-FEL:

a. Emittance Minimization in Photoinjectors by Transverse and Longitudinal Laser Pulse Shaping

Flexible control of the laser pulse distribution can significantly improve the performance of a photocathode. For example, a longitudinally uniform laser pulse will not produce a uniform electron pulse because the electron self field and Schottky effect will lead to a quantum efficiency drop, which is dependent on the laser arrival time. Having available a programmable transverse laser profile will allow us to correct the nonuniformity of the QE distribution.

The DUV-FEL is uniquely qualified to carry out an experimental demonstration of emittance minimization by laser pulse shaping. The Ti-Sapphire laser system (100 fs, $50 \mathrm{~mJ})$ at the DUVFEL possesses the bandwidth required for longitudinal pulse shaping. The experience of working on high-QE magnesium cathodes at BNL will give us great flexibility to produce a large range of charge densities. Furthermore, the extensive beam instrumentation available will allow us to characterize the electron beam.

\section{b. Ballistic Compression of High Brightness Electron Beams}

An rf photocathode injector is not only capable of optimizing the transverse emittance, but also the longitudinal emittance, if the proper charge and $\mathrm{rf}$ gun launch phase are chosen, i.e., the 6dimension phase space of the electron beam can be optimized. An electron beam is first generated by a relatively long laser pulse ( $8 \mathrm{ps}, \mathrm{FWHM})$, and it is continuously compressed as the beam energy increases. The electron beam is compressed in three stages, first in the rf gun, then in the succeeding drift space, followed by off-crest acceleration in the linac.

c. Electron Bunch Breakup Due to Coherent Synchrotron Radiation (CSR) and Space Charge Effects in Magnetic Bunch Compressors

Electron beam microbunching during the bunch compression has been observed at the DUV-FEL facility for several years, and significant progress was made recently in both theoretical understanding and experimental characterization. Several possible sources have now been 
identified: photocathode rf gun drive laser structure, longitudinal space-charge effects, and CSR. The DUV-FEL is one of a handful of facilities in the world capable of tackling these challenging scientific issues in a timely fashion.

\section{d. Electro-Optical Bunch Length Detector}

By taking advantage of the HGHG seed laser, we have designed and constructed a femtoseconds electro-optical bunch length detector at the DUV-FEL facility. We intend to continue to enhance the performance of this detector in both the electron beam bunch length and timing jitter characterization, and understand the ultimate resolution limit.

\section{LANL (Bruce Carlsten, LANL)}

Los Alamos has two high-brightness photoinjector electron beam facilities that have been used for beam physics R\&D research, and which would be capable of advanced research on the production of bright electron beams. Both facilities would be classified as integrated $\mathrm{rf}$ gun/linac or photoinjector designs, with a solenoid providing focusing and emittance compensation just after the cathode, and with several accelerating cavities operating as a single rf standing-wave structure.

The SPA facility is a $7.5-\mathrm{MeV}, 5.5$-cell photoinjector operating at $1.3 \mathrm{GHz}$. Typical operating parameters are bunch charges of $1 \mathrm{nC}$ and bunch lengths from 5 to $20 \mathrm{ps}$, with rms emittances as low as $2.5 \mathrm{~mm}$-mrad. This facility has a 4-dipole chicane, which has been used to compress the beam down to subpicosecond FWHM lengths. Diagnostics include a spectrometer, OTR screens, and electrically coupled beam position monitors, which have been used to measure beam emittance using Miller's technique.

The second facility is the AFEL, which is a $20-\mathrm{MeV}, 10.6$-cell photoinjector, also operating at $1.3 \mathrm{GHz}$. The photoinjector design included several advanced $\mathrm{rf}$ concepts, including minimization of quadrupole rf fields due to cavity-to-cavity coupling. Typical operating parameters are bunch charges from 1 to $5 \mathrm{nC}$, with bunch lengths from 5 to $20 \mathrm{ps}$ and rms emittances as low as $2 \mathrm{~mm}$-mrad (slice emittances as low at $1.5 \mathrm{~mm}$-mrad). This facility includes the necessary optics (beam and light) for making an FEL, and has been used for amplifier FEL, SASE FEL, and coherent transition radiation experiments. Associated with the AFEL is a cathode fabrication station, which is currently doing research on cesium telluride and cesium potassium antimonide cathodes, for improving lifetime and ruggedness of these cathodes.

\section{LBNL (John Corlett, LBNL)}

Facilities available at LBNL to support high-brightness electron sources and beam conditioning studies include modeling codes and expertise in their use; an rf and microwave test laboratory with vector network analyzers, spectrum analyzers, and sampling oscilloscopes for hardware measurements; laser systems expertise and hardware; low-level rf and controls expertise. Experience with modeling codes includes 3-D MAFIA electromagnetic software with PIC capability, PARMELA and ASTRA for particle beam dynamics, lattice codes MERLIN and MAD, and various codes for computation of collective effects. LBNL has experience and capabilities in design and fabrication of rf structures for high power applications, and in the 
design and construction of lattices for electron beams. LBNL staff are collaborators at the Fermilab FNPL photoinjector facility, with primary involvement in the development of emittance exchange techniques for production of a "flat" beam with large $\mathrm{x} / \mathrm{y}$ emittance ratio and small vertical normalized emittance, and are collaborating with a group at UCSC, Milan, in the development of cathodes for rf gun applications.

\section{MIT/Bates (Bill Graves, MIT)}

The MIT-Bates Linear Accelerator Center (see http://mitbates.Ins.mit.edu/bates) currently operates a $500-\mathrm{MeV}$ traveling wave $2856-\mathrm{MHz}$ electron linac with repetition rate up to $1 \mathrm{kHz}$. A single recirculation arc allows a second pass to a final energy of $1 \mathrm{GeV}$. The linac injector is a DC gun with strained GaAs photocathode driven by a diode laser for polarized beam production. The 20- $\mu \mathrm{A}$ linac beam is injected into the South Hall Ring where currents up to $200 \mathrm{~mA}$ are accumulated, then used for fixed target nuclear physics experiments. In addition to these facilities, there is a photoinjector test stand with electron and laser diagnostics that is operated independent of the larger accelerator.

The end of FY2005 marks a turning point for the Bates facility, where it is expected to complete its operation as a nuclear physics user facility. Two major roles are proposed for the future of the facility: the first is a Center for Accelerator Physics with a dual mission to educate the next generation of accelerator scientists and engineers and to engage in leading-edge accelerator technology development. The second role is as a site for an x-ray laser user facility (see http://mitbates.mit.edu/xfel).

Current Bates accelerator physics research activities include development of a method to drive lightly loaded CW superconducting rf structures with sub-kW power sources; stable, high-power rf modulator development for high-repetition-rate rf injectors; seeded FEL development; and design work on the proposed eRHIC collider at BNL. Bates is collaborating with the Quantum Electronics and Optics group at MIT to develop femtosecond synchronization of multiple lasers, and laser-to-rf timing. With the end of nuclear physics operations, the current infrastructure at Bates becomes available for accelerator physics development. This includes the photoinjector test stand; stable, high-power rf sources that operate at repetition rates above $1 \mathrm{kHz}$; and the accelerator structures themselves. Bates has substantial engineering staff and shops capable of cost-effective production of prototype accelerator equipment.

\section{SAIC/NRL (John Petillo, SAIC)}

The SAIC and NRL Team has facilities that include a suite of electromagnetic design tools, physicists and computational physicists, and laboratory facilities. This team has been engaged in a significant software research and development effort in support of vacuum electronics for over ten years. Their most recent intensive collaboration has provided a modeling and simulation code suite that includes the list of codes shown in Figure 3. 


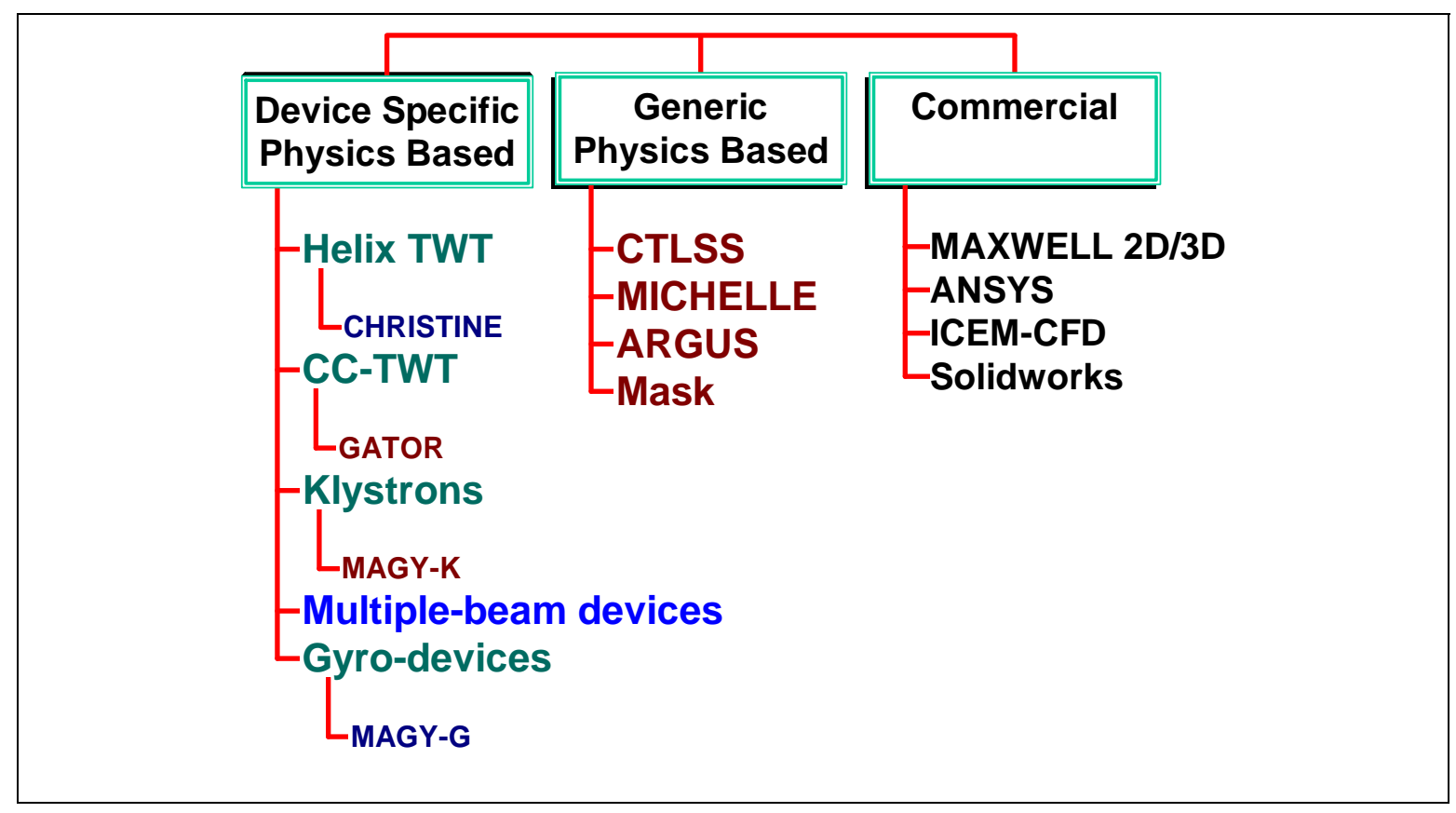

Figure 3: NRL vacuum electronics program design tools suite.

These codes range from first principles PIC and EM codes to semianalytic device-specific codes for rapid design and optimization. The codes include both finite-difference and modern finiteelement models. Basic code descriptions are as follows:

1. ARGUS (3D) and MASK (2D) Codes

- General-purpose time domain or frequency domain, electrostatic or electromagnetic, PIC

2. MICHELLE/Voyager

- 3D conformal-grid electron gun and collector modeling tool

3. CLTSS

- 3D frequency-domain EM code

4. CHRISTINE, CHRISTINE 3D

- Multifrequency large signal helix TWT codes

5. GATOR

- Time domain, CC-TWT code

6. MAGY

- Beam-circuit interaction model for fast and slow wave devices

The software development team includes 13 scientists and software engineers, and this team includes scientists from Los Alamos National Labs and Simulation Technology and Applied Research. This team is responsible for the software suite that NRL has built and continues to develop in support of vacuum electronic devices, including high-powered microwave devices and accelerator components. 
The combined NRL/SAIC facilities include the following high-performance parallel computer systems that are available for use:

SAIC:

40 - $1.0 \mathrm{GHz}$ Athlons - 0.5 GB per node - SCI interconnect

$8-1.8 \mathrm{GHz}$ Athlons - 2.0 GB per node - Miranet

$8-0.4 \mathrm{MHz}$ P-4 $\quad-0.5 \mathrm{~GB}$ per node - Ethernet

NRL:

8 - dual processor $1.8 \mathrm{GHz}$ Athlon CPUs - 4 GB RAM - gigabit Ethernet

128 - dual processor Xeon 2.2 GHz P4 - 1.0 GB per node - Miranet

- Machine is \#93 on the top 100 machines in the world.

- SAIC architected this machine and should have access to it if needed

\section{SLAC/LCLS/GTF (David Dowell, SLAC)}

The SLAC Gun Test Facility (GTF) began operation in 1998 and was built to investigate beams from a 1.6-cell S-band photocathode gun for use as an electron source for the Linac Coherent Light Source (LCLS). The gun is a BNL/SLAC/UCLA gun III. The cathode material is either copper or magnesium, which allows operation with cathode fields as high as $120 \mathrm{MV} / \mathrm{m}$. The UV drive laser is Nd:glass capable of transform-limited pulse widths of $400 \mathrm{fs}$. The chirped pulse amplifier (CPA) system consists of a diode-pumped Time Bandwidth oscillator, a stretcher grating, a flash lamp pumped Positive Light regenerative amplifier, a compressor grating, and two BBO crystals to frequency-quadruple the 1.064 -micron light to $266 \mathrm{~nm}$, which is used to drive the cathode. The cathode is illuminated at normal incidence (the ATF gun at BNL uses grazing incidence). The gun and its solenoid (for emittance compensation) are followed by a 10 -ft SLAC section, a set of quadrupoles, view screens, and a dipole spectrometer. The quadrupoles and view screens are used to measure the projected emittance. The spectrometer is used to measure the energy, energy spread, the longitudinal emittance, and the slice emittance. The slice time resolution is $200 \mathrm{fs}$ or better. A new electro-optical diagnostic to measure the bunch shape and wakefields will be installed by the end of January 2004. This device uses the Pockels effect to measure the electron bunch's transient electric field using a $\mathrm{LiNbO}_{3}$ crystal and light from the drive laser.

\section{UCLA (Sven Reiche, UCLA)}

\section{Photoelectron Generated Amplified Spontaneous Radiation Source (PEGASUS)}

The initial goal of PEGASUS will be to test a novel photoinjector, the PWT photocathode gun. The PEGASUS plane wave transformer injector has been conditioned to $20 \mathrm{MW}$ of rf power. Initial operations show a $15-\mathrm{MeV}$ dark current beam that will be used for beam radiation studies. The design of a new $\mathrm{LaB}_{6}$ cathode will allow for both thermionic emission and photoinjection operation. Experiments currently planned include novel beam instrumentation, surface effects in optical transition radiation, and waveguide SASE FEL.

Pegasus Design Parameters:

Energy $\quad 12-18 \mathrm{MeV}$ 


$\begin{array}{ll}\text { Energy Spread } & 0.15 \% \\ \text { Emittance } & <4 \mathrm{~mm}-\mathrm{mrad} \\ \text { Bunch Charge } & 1 \mathrm{nC} \\ \text { Bunch Length } & 900 \text { microns } \\ \text { Beam Size } & 150 \text { microns }\end{array}$

\section{The Neptune Laboratory}

The Neptune photoinjector has been designed to produce optimized, emittance-compensated performance over a range of charges, expanding the potential of the laboratory to a wide range of possible experiments. This injector is combined with a linac to produce an output beam containing up to a nanocoulomb of charge at energies up to $15 \mathrm{MeV}$. While the bunch length of the photoinjector beam is fixed by the drive laser at $\sim 6 \mathrm{ps}$, bunches as short as $0.3 \mathrm{ps}$ have been measured after compression using a magnetic chicane or ballistic (rf) bunching. Possible advanced accelerator experiments at Neptune range from direct laser acceleration (with operation in the ultra-low charge and emittance mode) to IFEL and FEL microbunching (moderate charge and emittance) to monoenergetic PBWA and plasma wakefield acceleration using ultrashort, low-charge bunches. Beam physics experiments undertaken at Neptune have involved spacecharge emittance compensation, the effects of space charge on emittance measurements, beam compressibility, and emittance growth processes in bends. The Neptune beamline has also served as a laboratory for detailed comparison of beam parameters with a number of simulation codes, particularly the UCLA version of PARMELA.

$\begin{array}{ll}\text { Neptune Design Parameters: } \\ \text { Beam Energy } & 15 \mathrm{MeV} \\ \text { Energy Spread } & <0.5 \% \\ \text { Energy Jitter } & <1 \% \\ \text { Beam Size (rms) } & 100 \mu \mathrm{m} \\ \text { Beam Charge } & 300 \mathrm{pC} \\ \text { Bunch Length (rms) } & 4 \mathrm{ps} \\ \mathrm{CO}_{2} \text { Laser Power } & 200 \mathrm{GW} \\ \mathrm{CO}_{2} \text { Rayleigh Range } & 2.2 \mathrm{~cm} \\ \text { Pointing Jitter } & <100 \mu \mathrm{m} \\ \text { Alignment Precision } & <1 \mathrm{mrad}\end{array}$

\section{UM/NRL (Patrick O'Shea, U. of Maryland)}

\section{Photocathode Research (Experiment and Theory/Simulation)}

- This project is currently exploring the photoemission of commercially available cathodes (e.g., scandate, M-type, MM-type). The photoemissive properties of dispenser cathodes under laser illumination from IR to UV under applied fields of several $\mathrm{MV} / \mathrm{m}$, and their quantum efficiency and emission levels, are being quantified. The purpose of this effort is to provide experimental data to benchmark our cathode theory/simulation formulations, which will lead to the development of custom-engineered photocathodes for accelerator applications. 
- The theoretical program addresses multiple issues in the theory and simulation of photoemission, primarily from dispenser cathodes (traditionally a thermionic emitter) but also metallic needle cathodes (traditionally a field emitter). The primary tasks are devoted to developing theoretical models and simulation codes treating: (i) the nature of the emitter surface and its impact on thermally- and field-assisted photoemission; (ii) the characteristics of the emitted electron energy distribution and their relation to quantities important in electron beam generation and propagation (e.g., emittance); and (iii) a fundamental study of factors that modify the photoemission surface barrier in a manner affecting (and affected by) the previous efforts. A time-dependent simulation program to study the interaction of the laser with the dispenser cathode and to characterize photoemission from photons of energies both greater than and less than the work function, i.e., single- and multiphoton quantum efficiency, and a general theory to cover all wavelengths, is under development. The program is comprised of developing theoretical models, analyzing experimental data using those models, and developing a predictive time-dependent model to extrapolate present performance to conditions characteristic of advanced FELs.

\section{Injector Beam Dynamics (Experiment and Theory/Simulation)}

- This research effort is exploring the physics of space-charge-induced emittance growth in low-energy electron beams. Available test beds include the University of Maryland Electron Ring and various smaller test stands. Simulation codes include WARP (PIC code) and PARMELA. The UMER experimental program is characterized by a very close connection between experiments and simulations. Advanced diagnostic capabilities include timeresolved phase-space and energy-spread measurements (i.e., 6D phase space mapping). In the future, the beam dynamical efforts will be aligned to the photoemission studies to provide a comprehensive model that will predict the beam emittance $a b$ initio.

- Further details can be found at: http://www.ipr.umd.edu/ebte/ring/index.html

\section{NICADD/FNPL (Phillipe Piot, FNAL)}

\section{Existing Equipment and Capabilities}

The Fermilab/NICADD (Northern Illinois Center for Accelerator and Detector Development) Photoinjector Laboratory (FNPL) (see also http://nicadd.niu.edu/fnpl/) consists of a 11/2 cell Lband rf-gun equipped with a high quantum efficiency Cesium-Telluride photocathode allowing the photoemission of electron bunches with charge up to $\sim 15 \mathrm{nC}$ ). The bunches generated thereby are further accelerated up to $16 \mathrm{MeV}$ by a downstream superconducting TESLA cavity operating with a nominal accelerating gradient of $\sim 12 \mathrm{MV} / \mathrm{m}$. Downstream of the cavity the beamline includes a set of quadrupoles and steering dipole elements for beam focusing and orbit correction, a skew quadrupole channel that allows the generation of flat beam using an incoming angular-momentum-dominated beam, and a magnetic-bunch-compressor chicane that can enhance the bunch peak current up to approximately $2.5 \mathrm{kA}$. The diagnostics for measuring transverse beam properties consist of electromagnetic beam position monitors, optical transition radiation (or $\mathrm{YaG}$ ) screens (for measuring beam transverse density), and three emittance measurements stations based on the multislit mask technique. The bunch-length measurement is performed by a streak camera that streaks optical transition radiation pulses emitted by the 
bunch. An alternative frequency-domain bunch-length diagnostics based on Martin-Puplett interferometry of coherent transition radiation is also available.

Downstream of the beamline, the beam can be bent in a dispersive section to measure the beam energy distribution or transported in a user experimental area directly ahead.

The FNPL facility can be operated remotely; to date teams from LBNL and DESY have used this capability to remotely perform beam physics experiments.

\section{Current Activities}

Over the past months, our activities have mainly focused on demonstration of photoinjector production of flat beam with high transverse emittance ratio; our present goal is to reach emittance ratio of about 100 with charge per bunch of $0.5 \mathrm{nC}$.

The user experimental area presently includes a set of two experiments, installed by a team from UCLA and NIU, aiming to investigate (1) plasma wakefield acceleration of electrons and (2) the generation of $\sim 1-\mathrm{MeV}$ electron bunches based on a self-trapping mechanism using a steep plasma density transition.

\section{Future Plans}

The FNPL facility will soon (within one year from now) incorporate a second TESLA cavity (with an accelerating gradient of $35 \mathrm{MV} / \mathrm{m}$ ) thereby boosting the beam energy up to $\sim 50 \mathrm{MeV}$. At a later stage, two higher-harmonic cavities $(3.9 \mathrm{GHz})$ will also be installed. One of these cavities operates on the accelerating mode and will allow the linearization of the longitudinal phase space to allow for a more efficient magnetic-bunch compression. The other cavity operates on the dipole mode and will provide a unique diagnostic for time-dependent beam parameter measurements.

\section{Overseas Facilities}

\subsection{DESY (Klaus Flöttmann, DESY)}

The experimental $\mathrm{rf}$ gun development program at DESY is centered at the Photoinjector Test Facility in Zeuthen (PITZ) near Berlin. Construction of the facility started in the year 2000 in the framework of an international collaboration. Under the heading 'research and development on electron sources for FELs and linear colliders' detailed experimental studies of rf gun beam dynamics and benchmarking of simulation codes are performed in parallel with technical developments concerning high duty cycle operation, general reliability, cathode performance, diagnostics, tests of new laser concepts, etc. In its present set-up the test stand consists of a 1.6cell L-band rf gun and a long diagnostics section, and is complemented by a 5-MW klystron (upgrade to $10-\mathrm{MW}$ in preparation), a $\mathrm{Cs}_{2} \mathrm{Te}$ load-lock cathode system, a Nd:YLF laser, and general infrastructure. All components are compatible to the TESLA XFEL pulse structure. In the next year an upgrade of the beamline with a booster cavity, a second rf station, and extended diagnostics is foreseen. For details see: http://desyntwww.desy.de/pitz/ . 


\subsection{Frascati}

(Massimo Ferrario, INFN-LNF)

The SPARC project consists of a $150-\mathrm{MeV}$ advanced photoinjector aimed at investigating the generation of high-brightness electron beams and their compression via magnetic and/or velocity bunching. This beam will be used to drive a SASE-FEL visible-VUV experiment: this is aimed to investigate the problems related to the beam matching into a segmented undulator and the alignment with the radiation beam at $500 \mathrm{~nm}$, as well as the generation of nonlinear coherent higher harmonics.

The $150-\mathrm{MeV}$ photoinjector consists of a 1.6-cell $\mathrm{rf}$ gun operated at S-band $(2.856 \mathrm{GHz}$, of the BNL/UCLA/SLAC type) and high peak field on the cathode $(120 \mathrm{MeV} / \mathrm{m})$ with incorporated metallic photocathode $(\mathrm{Cu}$ or $\mathrm{Mg})$, generating a 6-MeV beam. The beam is then focused and matched into three SLAC-type accelerating sections, which boost its energy up to $150-200 \mathrm{MeV}$. The first section is embedded in solenoids in order to provide additional magnetic focusing to better control the beam envelope and the emittance oscillations. The photocathode drive laser is a Ti:Sa system with the oscillator pulse train locked to the rf. To perform temporal flat-top laser pulse shaping, we will manipulate frequency lines in the large bandwidth of Ti:Sa, either using a liquid crystal mask in the Fourier plane for a nondispersive optic arrangement or a collinear acusto-optic modulator (DAZZLER). We aim to achieve a pulse rise time shorter than $1 \mathrm{ps.}$

The photoinjector design is by now completed with full specification of each system component: all bids for acquisition of main components have been so far launched, so we expect to be on schedule with delivery of the rf gun, laser system, rf sources, and linac accelerating sections. The expected start of installation for the photoinjector components is confirmed for spring of 2005. The first beam at full energy is expected by the beginning of 2006 .

\subsection{Milan (John Corlett, LBNL)}

The laboratory of time resolved photoemission spectroscopy of the Dipartimento di Matematica e Fisica, Università Cattolica del Sacro Cuore, Brescia, Italy, has the following equipment: a Tisapphire amplified laser; two parametric oscillators for up-conversion $(800-180 \mathrm{~nm})$, down conversion (800-20000 nm), and compression of the pulse (to $20 \mathrm{fs}$ ); a UHV photoemission chamber with Auger-LEED; an ion gun; an e-beam evaporator; a 5-degrees-of-freedom LHe manipulator; a 5-degrees-of-freedom, 150- to $1500-\mathrm{K}$ manipulator; time of flight spectrometers; an interferometer; optical correlators; and a spectrometer.

The research activities are mainly focused on time-resolved photoemission processes from metals with the aim to study e-e and e-p scattering, nonlinear processes, image potential states, and fast electron dynamics at the metal surfaces. The aim is to provide a basic knowledge to allow design of optimal materials for photoinjectors suitable for producing electron bunches with high charge density, very low emittance, and on the time scale of fs-ps for FEL and recirculating 3- to 4-GeV machines. A collaboration between LBNL and Prof. Fulvio Parmigiani of UCSC has being formed to study the physics and materials science of photocathodes and to develop an optimized and reliable photocathode system. A study of the following aspects of the physics of the photoemission process will be undertaken: 
Measurements on $\mathrm{Cu}, \mathrm{Ag}$, and other candidate photocathode materials

o Reflectivity for near-normal incidence at UV wavelengths

o Quantum yield (intrinsic quantum efficiency)

o Angle-resolved photoemission spectrum

o Lifetime measurements (day/week/month time-scales)

o Bulk and thin-film surface morphology and metrology

Theory and calculation of linear photoemission processes in semiconductors and metals

o Monte Carlo simulation of the photoemission process

o Band-gap engineering

o Model building and comparison with experiment

o Simulation of TOF spectrometer measurements

Design for equipment suitable for material studies and simulation of new photocathodes and if guns for advanced photoinjectors 


\section{Appendix B - Program of the ANL Theory Institute on Production of Bright Electron Beams}

Web page: http://www.aps.anl.gov/asd/theory/

\section{Monday - Argonne Guest House, Conference Room A}

8:20 Coffee and pastries

8:50 Greetings

9:00 General Session G1 - Chair, P. O'Shea

Introductory remarks

Review of experiments on bright e-beam production

Summary of the Berlin WS

H. Grunder

K.-J. Kim

P. Piot

J. Lewellen

10:30 Coffee break

11:00 Topical Session A: Emission Processes - Chair, K. Jensen

Survey of the emission process

K. Jensen

Virtual cathode effects

P. O'Shea

Measurement of thermal emittance

W. Graves

Discussion

12:30 Lunch

1:30 Topical Session A continues

3:00 Coffee break

3:30 Topical Session A continues

6:00 Pizza and Beer reception at K.-J. Kim's home

\section{Tuesday - Building 401, Conference Room A5000}

8:30 Coffee and pastries

9:00 General Session G2 - Chair, J. Galayda

FEL scaling and super-bright electron beams

What I learned from 15 years of rf gun operation at BNL

M. Zolotorev

Compact wakefield measurement facility

X. Wang

J. Power

10:30 Coffee break

11:00 Topical Session B: Beam Manipulation - Chair, G. Stupakov

Conditioned beams: principles and consequences
A. Sessler
S. Reiche
G. Stupakov

Effects due to strong focusing lattice

Limitations of a strong conditioner

12:30 Lunch 
1:30 Topical Session B continues

Design of a beam conditioner

A. Wolski

Two Laser-Plasma Methods for Conditioning Beams

A. Sessler

Further remarks

K.-J. Kim

Discussion

3:00 Coffee break

3:30 Topical Session B continues

\section{Wednesday - Building 401, Conference Room A5000}

8:30 Coffee and pastries

9:00 General Session G3 - Chair, W. Gai

LCLS project

J. Galayda

APS injector test stand

J. Lewellen

10:00 Topical Session C: RF Photocathode Beam Dynamics - Chair, K. Flöttmann Emittance Compensation Overview

B. Carlsten

10:30 Coffee break

11:00 Topical Session C continues

Beam dynamics in rf photocathode gun

M. Ferrario

Microbunching

W. Graves

Virtual cathode and longitudinal phase space

D. Dowell

12:30 Lunch

1:30 Topical Session C continues

Longitudinal instabilities space charge in photoinjector

C. Limborg

beamlines

Discussion

3:00 Coffee break

3:30 Topical Session C continues

6:30 No-host dinner at the Greek Islands Restaurant, Lombard 


\section{Thursday - Building 401, Conference Room A5000}

8:30 Coffee and pastries

9:00 General Session G4 - Chair, X. Wang

LEUTL-ALFF project

S. Milton

Photoinjector guns at AWA

M. Conde

10:00 Topical Session D: R\&D Planning - Chair, A. Sessler

Discussion of DOE 20-year road map

J. Corlett

10:30 Coffee break

11:00 Topical Session D continues

LCLS gun development

Gun development at Advanced Energy Systems

D. Dowell

Discussion

H. Bluem

12:30 Lunch

1:30 Topical Session D continues

3:00 Coffee break

3:30 Topical Session D continues

Friday - Building 401, Conference Room A5000

8:30 Coffee and pastries

9:00 Summary session - Chair, K.-J. Kim

Emissions Processes

K. Jensen

Beam Manipulation

G. Stupakov

RF Photocathode Beam Dynamics

K. Flöttmann

10:30 Coffee break

11:00 Summary session continues

R\&D Planning

Closing remarks

A. Sessler

K.-J. Kim

12:00 Meeting adjourns 


\section{Appendix C - List of Participants at the ANL Theory Institute on Production of Bright Electron Beams}

Hans Bluem

Advanced Energy Systems

P.O. Box 7455

Princeton, NJ 08543-7455

E-mail: bluem@grump.com

Phone: 609-514-0315

Fax: 609-514-0318

Bruce Carlsten

Los Alamos National Laboratory

P.O. Box 1663

Los Alamos, NM 87545

Shipping Address: Bikini Atoll Rd., SM30

E-mail: bcarlsten@lanl.gov

Phone: 505-667-5657

Fax: 505-667-8207

Manoel Conde

Argonne National Laboratory

HEP 362

9700 South Cass Avenue

Argonne, IL 60439

E-mail: conde@hep.anl.gov

Phone: 630-252-6099

Fax: 630-252-5076

John Corlett

Lawrence Berkeley National Laboratory

Center for Beam Physics

MS71-259

1 Cyclotron Road

Berkeley, CA 94720

E-mail: jncorlett@lbl.gov

Phone: 510-486-5228

Fax: 510-486-7981
David H. Dowell

Stanford Linear Accelerator Center

A/D Sources \& Polarization 04H

2575 Sand Hill Road, MS-18

Menlo Park, CA 94025

E-mail: dowell@slac.stanford.edu

Phone: 650-926-2494

Fax: 650-926-8533

Massimo Ferrario

Divisione Acceleratori

INFN-LNF

Via E. Fermi 40

Frascati, ROMA 00044

Italy

E-mail: ferrario@lnf.infn.it

Phone: 390694032216

Fax: 390694032265

Klaus Flöttmann

DESY

Notkestrasse 85

22603 Hamburg

Germany

E-mail: klaus.floettmann@desy.de

Phone: 49-40-8998-2052

Wei Gai

Argonne National Laboratory

HEP 362

9700 South Cass Avenue

Argonne, IL 60439

E-mail: wg@hep.anl.gov

Phone: 630-252-6560 
John N. Galayda

Linac Coherent Light Source

SLAC

2575 Sand Hill Road, MS-69

Menlo Park, CA 94025

E-mail: galayda@slac.stanford.edu

Phone: 650-926-4100

Rodney Gerig

Argonne National Laboratory

ASD 401

9700 South Cass Avenue

Argonne, IL 60439

E-mail: rod@aps.anl.gov

Phone: 630-252-5710

Fax: 630-252-7369

William Graves

Massachusetts Institute of Technology

BATESLINAC

77 Massachusetts Avenue

Cambridge, MA 02139-4307

E-mail: wsgraves@mit.edu

Phone: 617-253-9260

Katherine Harkay

Argonne National Laboratory

ASD 401

9700 South Cass Avenue

Argonne, IL 60439

E-mail: harkay@aps.anl.gov

Phone: 630-252-9758

Fax: 630-252-4732
Kevin L. Jensen

Naval Research Laboratory

Code 6841, ESTD, NRL

Washington, DC 20375-5347

E-mail: kevin.jensen@nrl.navy.mil

Phone: 202-767-3114

Fax: 202-767-1280

Kwang-Je Kim

Argonne National Laboratory

ASD 401

9700 South Cass Avenue

Argonne, IL 60439

E-mail: kwangje@aps.anl.gov

Phone: 630-252-4647

Fax: 630-252-7369

John Lewellen

Argonne National Laboratory

ASD 401

9700 South Cass Avenue

Argonne, IL 60439

E-mail: lewellen@aps.anl.gov

Phone: 630-252-5252

Fax: 630-252-4732

Yuelin Li

Argonne National Laboratory

ASD 401

9700 South Cass Avenue

Argonne, IL 60439

E-mail: ylli@aps.anl.gov

Phone: 630-252-7863

Fax: 630-252-4732 
Cecile Limborg

SSRL/Stanford Linear Accelerator Center

Mail Stop 69

2575 Sand Hill Road

Menlo Park, CA 94025

E-mail: limborg@slac.stanford.edu

Phone: 650-926-8685

Steve Milton

Argonne National Laboratory

XFD 401

9700 South Cass Avenue

Argonne, IL 60439

E-mail: milton@aps.anl.gov

Phone: 630-252-9101

Patrick G. O'Shea

Institute for Research in Electronics \&

Applied Physics

University of Maryland

Energy Research Building

College Park, MD 21054-3511

E-mail: poshea@umd.edu

Phone: 301-405-4977

John Petillo

Science Applications International Corporation Suite 130

20 Burlington Mall Road

Burlington, MA 01803

E-mail: jpetillo@bos.saic.com

Phone: 781-221-7615

Fax: 781-270-0063
Philippe Piot

Fermi National Accelerator Laboratory

BD-A0 Photo Injector Group

MS - 306

Box 500

Batavia, IL 60510

E-mail: piot@fnal.gov

Phone: 630-840-6389

Fax: 630-840-8248

John Power

Argonne National Laboratory

HEP 362

9700 South Cass Avenue

Argonne, IL 60439

E-mail: jp@anl.gov

Phone: 630-252-3191

Sven Reiche

Department of Physics \& Astronomy

University of California-Los Angeles

4-166B Knudsen Hall

Los Angeles, CA 90095-1547

E-mail: reiche@physics.ucla.edu

Phone: 310-206-4540

Fax: 310-825-8432

Andy Sessler

Lawrence Berkeley National Laboratory Accelerator \& Fusion Research (AFCB35)

1 Cyclotron Road

MS 71R0259

Berkeley, CA 94720

E-mail: amsessler@lbl.gov 
Gennady V. Stupakov

Stanford Linear Accelerator Center

ARD-A Collective Effects (02D)

2575 Sand Hill Road, MS-26

Menlo Park, CA 94025

E-mail: stupakov@slac.stanford.edu

Phone: 650-926-4320

Fax: 650-926-5368

Chun-xi Wang

Argonne National Laboratory

ASD 401

9700 South Cass Avenue

Argonne, IL 60439

E-mail: wangcx@aps.anl.gov

Phone: 630-252-4968

Xijie Wang

Brookhaven National Laboratory

Building 725C

Upton, NY 11973-5000

E-mail: wangx@bnl.gov

Phone: 631-344-5791

Fax: 631-344-3029

Andrzej Wolski

Lawrence Berkeley National Laboratory

1 Cyclotron Road

Mail Stop 71R0259

Berkeley, CA 94720

E-mail: AWolski@lbl.gov

Phone: 510-486-6549

Fax: 510-486-6485
Max Zolotorev

Lawrence Berkeley National Laboratory

Accelerator \& Fusion Research (AFCB45)

1 Cyclotron Road, MS 71R0259

Berkeley, CA 94720

E-mail: max_zolotorev@lbl.gov 